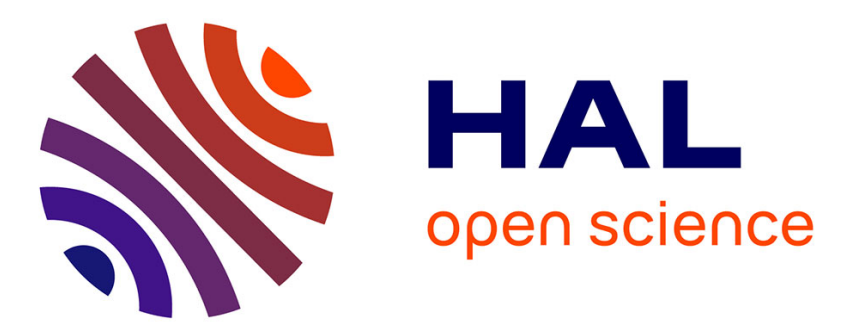

\title{
Computational and experimental investigation of flow over a transient pitching hydrofoil
}

\author{
Antoine Ducoin, Francois Deniset, Jacques-André Astolfi, Jean-François
}

Sigrist

\section{- To cite this version:}

Antoine Ducoin, Francois Deniset, Jacques-André Astolfi, Jean-François Sigrist. Computational and experimental investigation of flow over a transient pitching hydrofoil. European Journal of Mechanics, 2009, 28 (6), pp.728-743. 10.1016/j.euromechflu.2009.06.001 . hal-01206317

\section{HAL Id: hal-01206317 \\ https://hal.science/hal-01206317}

Submitted on 28 Sep 2015

HAL is a multi-disciplinary open access archive for the deposit and dissemination of scientific research documents, whether they are published or not. The documents may come from teaching and research institutions in France or abroad, or from public or private research centers.
L'archive ouverte pluridisciplinaire HAL, est destinée au dépôt et à la diffusion de documents scientifiques de niveau recherche, publiés ou non, émanant des établissements d'enseignement et de recherche français ou étrangers, des laboratoires publics ou privés. 


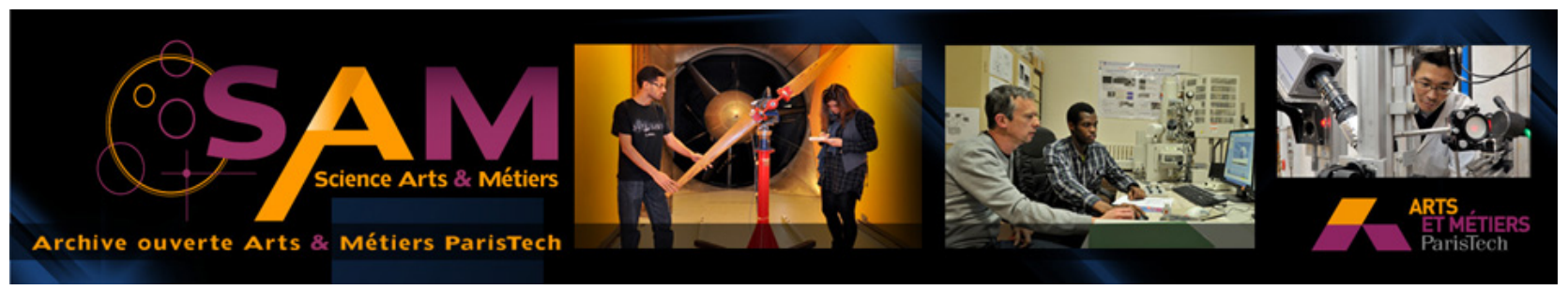

\section{Science Arts \& Métiers (SAM)}

is an open access repository that collects the work of Arts et Métiers ParisTech researchers and makes it freely available over the web where possible.

This is an author-deposited version published in: http://sam.ensam.eu

Handle ID: .http://hdl.handle.net/10985/10205

\section{To cite this version :}

Antoine DUCOIN, Francois DENISET, Jacques-André ASTOLFI, Jean-François SIGRIST Computational and experimental investigation of flow over a transient pitching hydrofoil European journal of mechanics - Vol. 28, nº, p.728-743 - 2009 


\title{
Computational and experimental investigation of flow over a transient pitching hydrofoil
}

\author{
Antoine Ducoin ${ }^{a, *}$, François Deniset ${ }^{a}$, Jacques André Astolfi ${ }^{a}$, \\ Jean-François Sigrist ${ }^{b}$ \\ ${ }^{a}$ Institut de Recherche de l'Ecole Navale EA 3634, Ecole Navale, 29240 Brest Armées, \\ France \\ ${ }^{b}$ DCNS Propulsion, 44620, La Montagne, France
}

\begin{abstract}
The present study is developed within the general framework of marine structure design of lifting bodies, operating in transient regimes. The study concerns the experimental and numerical investigations of time-space distribution of the wall pressure field on a NACA66 hydrofoil in a forced pitching motion from $0^{\circ}$ to $15^{\circ}$ beyond stall for four pitching velocities. Experiments in a hydrodynamic tunnel and corresponding RANS calculations are carried out. Wall pressure transducers are located along the chord on the suction side of the hydrofoil. The numerical approach is conducted in transient regime using a recent laminar to turbulent transition model. First, the flow is analyzed on the basis of the pressure fluctuations to highlight the laminar to turbulent transition. The evolutions of transition, laminar bubble and leading and trailing edge detachments as well as pressure fluctuations are discussed for various transient pitching motions. Then, hydrodynamic coefficients are analyzed in order to quantify the transient effects on hydrodynamic
\end{abstract}

\footnotetext{
*corresponding author

1 antoine.ducoin@ecole-navale.fr
} 
loading. The comparison of calculated and measured local wall pressures on the suction side leads to the identification of the effect of pitching velocity on hydrodynamic loading.

Key words: Lifting bodies; hydrodynamic loading; transient regimes; laminar to turbulent transition

\section{INTRODUCTION}

The prediction of the loading of lifting bodies such as rudders, stabilizers or marine propellers operating in forced motions of large amplitude is fundamental in the context of marine design. It requires a good understanding of phenomena such as transition, turbulence and stall [1]. It includes the boundary layer study in unsteady forced regime which has been the object of many researches, including the prediction by RANS based codes in the context of industrial applications. Srinivasan et al. (1995) [2] and Barakos (2000) [3] show the accuracy of RANS codes to predict hydrodynamic loading of foils in cases of low angles of incidence, and highlight the turbulence model dependency when separation is strong. The influence of pitching velocities can be also of primary importance on loading prediction in the case of low Reynolds numbers. Hamadi et al. (2000) [4] show that inertial effects are increasing with pitching velocities. The authors include non-dimensionalized parameters useful in transient regimes. An experimental study is presented in [5] for an oscillating airfoil at various reduced frequencies $\kappa=\omega c / 2 U_{\infty}$ (with $\omega$ the pitching velocity) and at $R e=1.35 \times 10^{5}$. It helps to study the effect of pitching velocity. For small reduced frequencies, boundary layer events produce variations in lift, drag and moment coefficients. As well, 
boundary layer transition caused by laminar separation is delayed and promoted when reduced frequencies increases. The lift coefficient and lift-curve slope have also some modifications but it was shown that the laminar bubble length is insensitive to reduced frequency. Recent works focus on the impact of transition modeling in RANS based codes $[6,7,8]$. It has an impact on stall and loading prediction. Smith et al. (2004) [9] show that fully turbulent computations over predict lift and drag. Shelton et al. (2005) [6] include a transition model and show its impact on hydrodynamic coefficients.

The specific case of separation induced transition has been studied in many works concerning aerodynamic applications. The transition is caused by a recirculation zone induced by a laminar separation. It is well known that after the boundary layer transition to turbulence, the flow reattaches. Tani (1964) [10] showed that the bubble length is reduced when the pressure gradient increases because of the faster transition. Applied to foil geometry at significant angle of incidence, this induces a small bubble near the leading edge (as compared to the bubble length near the trailing edge for example at low angle of incidence) because of the higher pressure gradient created by the curvature. Concerning the prediction by RANS based codes, global effects are taken into account but smaller structures are not captured. Many works have been therefore considered on laminar separation bubble (LSB) by experimental methods (Gaster (1969) [11]) and DNS simulations [12, 13] and show that complex and multi scaled structures can occur in LSB wake. Based on Gaster works, Pauley et al. (1990) [14] studied an unsteady laminar separation bubble induced by an adverse pressure gradient on a leading edge geometry. It has been found that the frequency of the vortex shedding from 
the bubble can be non-dimensionalized with a Strouhal number $S_{t \theta}$ based on momentum thickness $(\theta)_{\text {sep }}$ and external velocity $\left(u_{e}\right)_{\text {sep }}$ at laminar separation which appears in many recent works $[15,16]$. Moreover, some recent numerical studied of DNS simulation have shown that those vortex shedding resulting into a flapping motion of the bubble generates some velocity and pressure fluctuations backward the bubble, see Rist and Maucher (2002) [17] in the case of a flat plate and Jones et al. (2008) [18] in the case of an airfoil .

The present paper focuses on the spatio-temporal evolution of the wall pressure field of an hydrofoil arising from transient pitching motion at $R e=$ $0.75 \times 10^{6}$. Both experimental and numerical approaches were developed The experiment consist in measuring the wall pressure using pressure transducers at several points located along the chord on the suction side of the hydrofoil. Computations are led with the CFD RANS based code $C F X{ }^{\complement}$. The first aim of this study is a better understanding of hydrodynamic loading responses on boundary layer events like transition and laminar separation induced transition. Another challenging task is to check the accuracy and the limitations of the RANS simulations to predict those phenomena.

The flow is first studied in the case of a low pitching velocity. Measured and computed wall pressures near the leading edge and the trailing edge help to characterize separation and transition localization on the hydrofoil. Measured pressure fluctuations are analyzed on those transducers using spectrograms. The influence of the pitching velocity on the boundary layer events is investigated. Hydrodynamic coefficients are analyzed and dynamic effects of the pitching velocity on hydrodynamic loading are highlighted. 


\section{EXPERIMENTAL SET UP}

Measurements are carried out in the cavitation tunnel at IRENav. The test section is $1 \mathrm{~m}$ long and has a $0.192 \mathrm{~m}$ square section. It allows the authors to control the velocity range between 0 and $15 \mathrm{~m} / \mathrm{s}$ and the pressure range from 30 mbar to 3 bars. The hydrofoil is a NACA 66 witch presents a camber type NACA $a=0.8$, a camber ratio of $2 \%$ and a relative thickness of $12 \%$ [19]. It is mounted horizontally in the tunnel test section. The chord is $c=0.150 \mathrm{~m}$ and the span is $b=0.191 \mathrm{~m}$.

Pressure measurements are carried out using seventeen piezo-resistive transducers (Keller AG 2 MI PAA100-075-010) of 10 bars maximum pressure. The pressure transducers are mounted into small cavities with a $0.5 \mathrm{~mm}$ diameter pinhole at the hydrofoil surface. The wall pressure spectrum measured by the transducer is attenuated from the theoretical cut off frequency $f_{c}=9152 \mathrm{~Hz}$. Experiments are led with a sample frequency of $f=20 k H z$.

The transducer locations are given in Figure 1. As shown, one set of ten transducers is aligned along the chord on the suction side at mid span, starting from the leading edge at reduced coordinate $x / c=0.1$ up to the trailing edge at coordinate $x / c=0.90$ with a step of $0.10 c$. Two sets of three transducers are arranged parallel to this line in order to analyze three-dimensional effects, which is beyond the scope of this paper. A study of those $3 \mathrm{D}$ effects is presented in Leroux et al. (2005) [19]. As well, the transducer located at the pressure side is not useful in this study as long as we are interested on suction 
side.

The paper presents some measurements in which a roughness patch has been added near the leading edge. This allows us to suppress the transition to turbulence zone and Laminar Separation Bubble. The patch has $16 \mu$ grain and is $250 \mu$ thick.

The nominal free stream velocity $U_{\infty}$ is $5 \mathrm{~m} / \mathrm{s}$, corresponding to a Reynolds number based on the foil chord length of $R e=0.75 \times 10^{6}$. The uncertainty for the free stream velocity is $\Delta U_{\infty}= \pm 0.02 \mathrm{~m} / \mathrm{s}$. The pressure in the tunnel test section was set to $P_{0}=1.4$ bars with an uncertainty of about $\Delta P_{0}= \pm 0.003$ bar. This pressure allow us to avoid cavitation which is not considered. The hydrofoil pitches about an axis located at $25 \%$ from the leading edge. The angle of incidence varies from $0^{\circ}$ to $15^{\circ}$ and then comes back to $0^{\circ}$, with at least 2 periods of acceleration and 2 periods of deceleration.

As shown in Figure 2, four pitching velocities are defined, from a considered low pitching velocity to a high pitching velocity.

The average rotation velocity is $\dot{\alpha}=2 \alpha_{\max } / t_{f}$, where $t_{f}$ is the total time of transient motion. Let's introduce a similarity parameter based on the chord length c and the upstream velocity $U_{\infty}$, which gives $\dot{\alpha}^{*}=\frac{\dot{\alpha} \times c}{U_{\infty}}$.

In some cases, the pressure signal is decomposed using the "Empirical Mode Decomposition" (EMD, [20]). It has been the object of several hydrodynamic studies $[21,22]$. This method consists on the decomposition of an unsteady signal $x(t)$ into intrinsic oscillatory components called intrinsic mode functions (IMFs) by means of an algorithm called sifting process. The basic principle is the extraction of intrinsic time scale components of the 
signal starting from finer temporal scales (high frequency modes) to coarser ones (low frequency modes). The total sum of the extracted IMFs matches the signal and therefore ensures signal complete reconstruction. Huang et al.(1998) [20] have introduced the EMD method for analyzing data from unsteady and nonlinear processes. In the present paper, the method is used first for the reconstruction of a low frequency signal $\bar{x}(t)$ in order to obtain a trend signal. This permits a more pertinent comparison with computations which are resolved with RANS equations. An other aim is to use the medium and high frequency components to reconstruct a signal $\widetilde{x}(t)$ in order to analyze the pressure fluctuations with spectrograms. Then the reconstructed signal can be written as [23]:

$$
\begin{gathered}
\bar{x}(t)=\sum_{j=k}^{n} I M F_{j}(t)+r_{n}(t) \\
\widetilde{x}(t)=\sum_{j=1}^{k-1} I M F_{j}(t)
\end{gathered}
$$

where $n$ is the number of modes and $r_{n}(t)$ is the residue [20].

An example is shown in Figure 3 where the reconstructed pressure signal $\overline{C_{P}}(t)$ corresponds to low frequency modes whereas the reconstructed pressure signal $\widetilde{C_{P}}(t)$ corresponds to medium and high frequency modes for $k=10$.

Experimental frequencies resulting from wall pressure fluctuations are then non dimensionalized using the dimensionless shedding frequency of Pauley et al. (1990) [14]. It is based on boundary layer momentum thickness $(\theta)_{\text {sep }}$ and local free stream velocity $\left(u_{e}\right)_{\text {sep }}$ at separation point which are determined experimentally and it can be written as $S_{t \theta}=\frac{f(\theta)_{s e p}}{\left(u_{e}\right)_{s e p}}$. In 
this paper the Strouhal number is computed by numerical values of $(\theta)_{\text {sep }}$ and $\left(u_{e}\right)_{\text {sep }}$ and experimental value of the frequency. It's then compared with the Strouhal number $S_{t \theta}=0.00686[14]$.

\section{FLOW MODELING AND NUMERICAL RESOLUTION}

The fluid problem is solved with the finite volume technique using the CFD code $C F X^{\text {C }}$. The fluid flow is described by the mass and momentum conservation equations which read for an incompressible and viscous fluid:

$$
\begin{gathered}
\frac{\partial v_{j}}{\partial x_{j}}=0 \\
\frac{\rho \partial\left(v_{i}\right)}{\partial t}+\frac{\rho v_{j} \partial\left(v_{i}\right)}{\partial x_{j}}=-\frac{\partial p}{\partial x_{i}}+\mu \frac{\partial^{2} v_{i}}{\partial x_{j} \partial x_{j}}
\end{gathered}
$$

where $v$ is the fluid velocity, $\rho$ is the fluid density, $p$ is the pressure and $\mu$ is the dynamic viscosity.

Equations of mass and momentum are integrated over a control volume $\Omega_{F}$ of boundary $\partial \Omega_{F}$, using the Leibnitz rule and the Gauss theorem. The general form of an integrated conservation equation for a scalar fluid unknown $\Phi$ is the following one:

$$
\frac{\partial}{\partial t}\left(\int_{\Omega_{F}} \rho_{F} \phi d \Omega_{F}\right)+\int_{\partial \Omega_{F}} \rho_{F} v_{j} \phi d n_{j}=\int_{\Omega_{F}} S_{\phi} d \Omega_{F}+\int_{\partial \Omega_{F}} \Gamma \frac{\partial \phi}{\partial x_{j}} d n_{j}
$$

The time dependent terms are approximated by a second order backward Euler scheme:

$$
\frac{d}{d t}\left(\int_{\Omega_{F}} \rho_{F} \phi d \Omega\right) \approx \frac{\rho_{F}\left|\Omega_{F}\right|}{\delta t}\left(3 / 2 \phi_{P}^{n+1}-2 \phi_{P}^{n}+1 / 2 \phi_{P}^{n-1}\right)
$$


where $n, n-1$ and $n+1$ are the time steps.

The convective and diffusive terms $(C$ and $D)$ are calculated using finite difference approximations, leading to the global expression :

$$
\int_{\partial \Omega_{P}(t)} \rho v_{j} \phi-\Gamma \frac{\partial \phi}{\partial x_{j}} d n_{j} \approx \sum_{M} C_{M}^{n+1} \phi_{M}^{n+1}-D_{M}^{n+1} \phi_{M}^{n+1}
$$

where $M$ stands for the neighboring points of cell $\Omega_{P}$. Nodal values are computed with a high resolution upwind scheme. This advection scheme is implemented into the CFD code and can be cast in the form:

$$
\phi_{p}=\phi_{u p}+\beta \nabla \phi \Delta \vec{r}
$$

where $\phi_{p}$ and $\phi_{u p}$ are respectively the values of $\phi$ at the integration point $P$ and at the upwind node (depending on the flow direction). $\beta$ is a relaxation coefficient ranging between 0 and 1. The High resolution scheme computes $\beta$ locally in order to be as close to 1 as possible without violating boundedness principles, see Barth and Jesperson (1989) [24]. $\vec{r}$ is the vector from the upwind node to the integration point $P$. A value of $\beta=1$ leads to a second order upwind difference scheme whereas a value of $\beta=0$ leads to a first order upwind difference scheme. Taking into account Equations (7) and (8) leads to the following algebraic non-linear system:

$$
A_{P} \phi_{P}^{n+1}=\sum_{M} A_{P} \phi_{P}^{n+1}+b_{P} \phi_{P}^{n}
$$

\subsection{Turbulence and transition modeling}

The calculations are performed with the CFD RANS based code $C F X^{\complement}$. The $k-\omega S S T$ model appears to be an accurate turbulence model for bound- 
ary layer detachment prediction [25, 26, 27].

The $k-\omega S S T$ turbulence model is coupled with a transition model $\gamma-\operatorname{Re}_{\theta}$ which uses experimental correlations based on local variables [28, 29, 30]. The model is based on two transport equations. The first one is for intermittency $\gamma$ which triggers the transition process:

$$
\frac{\partial(\rho \gamma)}{\partial t}+\frac{\partial\left(\rho v_{j} \gamma\right)}{\partial x_{j}}=P_{\gamma}+E_{\gamma}+\frac{\partial}{\partial x_{j}}\left[\left(\mu+\frac{\mu_{t}}{\sigma_{f}}\right) \frac{\partial \gamma}{\partial x_{j}}\right]
$$

where $P_{\gamma}$ and $E_{\gamma}$ are the transition sources based on empirical correlations [28]. $\mu_{t}$ is the friction velocity and $\sigma_{f}$ is a constant.

The second one is a transport equation for the transition momentum thickness Reynolds number $\overline{\operatorname{Re} \theta_{t}}$ is given by:

$$
\frac{\partial\left(\rho \overline{R e_{\theta t}}\right)}{\partial t}+\frac{\partial\left(\rho v_{j} \overline{R e_{\theta t}}\right)}{\partial x_{j}}=P_{\theta t}+\frac{\partial}{\partial x_{j}}\left[\sigma_{\theta t}\left(\mu+\mu_{t}\right) \frac{\partial \overline{R e_{\theta t}}}{\partial x_{j}}\right]
$$

with $P_{\theta t}$ a source term which forces $\overline{R e_{\theta t}}$ to match the local value of $R e_{\theta t}$ based empirical correlation. $\sigma_{\theta t}$ is a source term of diffusion control.

In this formulation, only local information is used to activate the production term in the intermittency equation. This model allows to capture major transition effects and is accurate in the case of separation induced transition. The intermittency is modified to accept values larger than 1 at separation in order to have a correct prediction of transition length. Complete transition model formulation is given in [30].

\subsection{Boundary conditions and discretization}

The 2D domain corresponds to the tunnel test section at IRENav. The ratio between the square section height $h$ and the chord length $c$ is $h / c=1.28$. 
The inlet velocity is set to $U_{\infty}=5 \mathrm{~m} / \mathrm{s}$ and the taken outlet reference pressure is set to zero. Symmetry conditions are set on top and bottom walls and a no slip condition is imposed on the hydrofoil surface. Transient computations are initialized with a stationary converged computation. As shown in Figure 4, the mesh is composed of 66,000 elements and 50 layers are used in the structured near wall zone. The other part of the domain is discretized with unstructured triangle elements. The boundary layer is discretized in order to satisfy $y^{+} \approx \frac{y u_{\tau}}{\nu}=1$. This ensures a low Reynolds resolution. Mesh refinements are performed at the leading edge, at the trailing edge and in the wake. The hydrofoil motion is taken into account using a changing boundary condition at the wall. To do that, foil mesh coordinates are calculated at each time step and the whole domain is then meshed again by moving each nodes. This technique uses a diffusivity parameter applied in the mesh displacement equation which induces a mesh stiffness [31, 32, 33]. This one is set to be inversely proportional to the wall distance in order to limit mesh distortion in the wall region. A view of the deformed mesh is shown in Figure 5 for the maximum angle of incidence $\alpha=15^{\circ}$. As shown, mesh distortion is small in the near wall region and cells have the same areas as the initial mesh, unless the normal to the wall have moved of about few degrees, see Figure 4. Far away from the hydrofoil, cells are highly extended near the top wall and highly compressed near the bottom wall. Moreover, the quality of the mesh stay very close to the quality of the initial mesh as shown by the evolution of the maximum expansion factor in Figure 6. The RANS equations are solved in an arbitrary referential with the Arbitrary Lagrangian Euler formulation (ALE) [32]. 
Mesh convergence is carried out on hydrodynamic coefficients for an angle of attack of $6^{\circ}$ and a steady flow. Table 1 summarizes the lift and drag coefficients for each tested mesh case. The thickness of the structured mesh near the wall and the aspect ratio between structured and unstructured meshes have been kept. It appears that wall function predicts quite well the lift coefficient as compared to the low Reynolds resolution $y^{+}=0.3$ taken as a reference. On the other hand, the wall functions under predict the drag coefficient of about $15 \%$. This is due to the contribution of wall shear to the drag coefficient. The influence of the number of elements has been investigated in Table 2. $N_{\text {foil }}$ is the number of nodes on hydrofoil's surface and $N_{\text {total }}$ is the total number of elements. The lift coefficient converges very fast, from $N_{\text {foil }}=100$. On the other hand, the drag coefficient converges from $N_{\text {foil }}=200$.

Temporal discretization has been set according to CFL number with fixed spatial mesh. Figure 7 shows pressure coefficients located at $x / c=0.1$ versus time for pitching motion from $0^{\circ}$ to $15^{\circ}$ and $\dot{\alpha}^{*}=0.18$. We focus on the non linear behavior show from $t=0.9 \mathrm{~s}$ to $t=1 \mathrm{~s}$. It appears to be associated to transition which needs a high temporal discretization level. Convergence is obtained for values lower than $\Delta t=0.001 s$. In the same way, Figure 8 shows the pressure coefficient when leading edge separation occurs. It is found to be very sensitive to $\Delta t$. For the lower $\Delta t$, there is an advance of separation whereas $\Delta t=0.001 s$ and $\Delta t=0.0005 s$ give same results. So, $\Delta t=0.001 s$ has been chosen for the pitching velocity of $\dot{\alpha}^{*}=0.18$. A minimum number of time steps is set to correctly take into account the dynamic in computation. To do that, the time step $\Delta t$ must verify 
$\frac{\Delta t}{t_{f}}>5 \times 10^{-4}$, with $t_{f}$, where $t_{f}$ is the total simulation time which depend of pitching velocity.

\section{RESULTS AND DISCUSSION}

Figure 9 (a) shows the measured pressure coefficient at $x / c=0.3$ during the transient motion from $0^{\circ}$ to $15^{\circ}$ for $\dot{\alpha}^{*}=0.18$ compared to the computed one. There is a good agreement between the experimental and the numerical results except for high frequency fluctuations which are not captured by the computation.

From $0^{\circ}$ to $5^{\circ}$, the pressure decreases with low fluctuations. At $5^{\circ}$, the pressure stops to decrease and shows an inflection with a high level of fluctuations. Then the pressure continues to decrease with significant fluctuations, increasing from $6^{\circ}$ to $13^{\circ}$. A more accurate analysis showed that the fluctuations are quasi-periodic and can be related to vortex shedding downstream a laminar separation bubble. From $13^{\circ}$ a strong pressure overshoot is observed, low frequency fluctuations with large amplitude are observed resulting of stall, as shown in Figure 9 (b). Again, the high frequency fluctuations are not capture by computation whereas the periodic peaks resulting from the leading edge vortex shedding are well reproduced. The relatively complex characteristic of the wall pressure evolutions comes from the various features of the boundary layer flow during foil rotation.

\subsection{Flow analysis}

The flow is first analyzed for the lowest pitching velocity $\dot{\alpha}^{*}=$ 0.18. Figure 10 shows the experimental and computed wall pressure coefficients evolutions for transducers located from $x / c=0.3$ to $x / c=0.9$. As 
shown, there is a good agreement between computations and measurements on wall pressures. A maximum difference of about $\Delta C_{P}=0.1$ is found at $x / c=0.9, x / c=0.7$ and $x / c=0.2$. Measured wall pressure coefficient at $x / c=0.9$ shows high level fluctuations which move and increase to $x / c=0.8$ at $\alpha \approx 2^{\circ}$ and then to $x / c=0.7$ at $\alpha \approx 4^{\circ}$ as shown in Figure 10 (b) and Figure 10 (c). Computations show a global increase of pressure coefficient without any fluctuation. We will see later that this is related to a LSB turbulent reattachment point passing from $\mathrm{x} / \mathrm{c}=0.8$ for $\alpha=2^{\circ}$ to $\mathrm{x} / \mathrm{c}=0.7$ for $\alpha=4^{\circ}$. This induces a strong temporal variation of pressure due to the constant pressure zone in the LSB region. For both computation and measurement, there is a net pressure inflection for $\alpha=5.7^{\circ}$ as transition is moving toward the leading edge. Figure 10 (d), (e) and (f) show that the measured pressure fluctuations highly increase up to a burst and continue to increase progressively to $\alpha=12^{\circ}$. Spatially, the level of fluctuations increases from $x / c=0.2$ to $x / c=0.5$. At the same time, wall pressure coefficient at $x / c=0.9$ and $x / c=0.8$ reaches a maximum value at respectively $\alpha=7^{\circ}$ and $\alpha=10^{\circ}$ compared to $\alpha=5.5^{\circ}$ and $\alpha=10^{\circ}$ according to computations. It corresponds to the trailing edge separation passing on the pressure transducers (Figure 12) for which the flow is decelerated and then accelerated by the reverse flow.

This experimental versus computation wall pressure analysis enables the authors to validate the simulation on local phenomena which events in the boundary layer like transition by laminar separation bubble and leading edge vortex shedding. The folowing results are from computations only and are assumed to correctly 


\section{reproduce the experimental flow.}

Figure 11 shows the velocity streamlines determined from computations. As shown at $0^{\circ}$, a reversed flow is located at $x / c=0.8$ resulting from a Laminar Separation Bubble (LSB) inducing a transition to turbulent flow at reattachment. The displacement of LSB towards the leading edge zone is observed up to $5^{\circ}$. At $5^{\circ}$ the LSB at trailing edge is replaced by a LSB at leading edge and stay close to this location when the angle of attack increase, as shown in Figure 11 for $\alpha=11^{\circ}$. Then, stall is observed for $13.6^{\circ}$ together with leading edge vortex shedding. At $\alpha=13.9^{\circ}$, a vortex extends along the suction side corresponding to the strong global pressure overshoot, as shown on Figure 9. Then two contra-rotative vortices are shed from the trailing edge $\left(\alpha=14.2^{\circ}\right)$. This scenario is repeated 3 times periodically in the case of the lowest pitching velocity. A reverse scenario is observed during downward rotation.

Separation and transition points have been located using the "wall shear stress equal to 0 criteria". The transition point is defined as the turbulent reattachment point [30]. Figure 12 summarizes the locations of the separations, reattachment and transition points from $\alpha=0^{\circ}$ to $13^{\circ}$ before stall. The vertical axis is the $x / c$ location along the chord from leading edge $(x / c=0)$ to trailing edge $(x / c=1)$. The trailing edge separation point is located very close to the trailing edge for $0^{\circ}$, and moves slowly towards the leading edge when the angle of incidence increases. For $\alpha=0^{\circ}$ to $5^{\circ}$ the two characteristic points (laminar separation and turbulent reattachment) are respectively located between $(x / c)_{\text {sep }}=0.74$ to 0.66 and $(x / c)_{\text {reattach }}=0.85$ to 0.69 . As a matter of fact, the global length of LSB tends to decrease as $\alpha$ increases. At 
these angles of incidences, the boundary layer around the hydrofoil is laminar. As shown on Figure 13, at $\alpha=4.7^{\circ}$, the trailing edge LSB disappears and a shorter LSB induced by higher pressure gradient appears at the leading edge which induces a shift of the transition location from $x / c=0.66$ to $x / c=0.08$ at $\alpha=5^{\circ}$. Then the boundary layer around the hydrofoil is fully turbulent. As well, the trailing edge separation $x_{T-E}$ shift from $x / c=0.99$ to $x / c=0.92$ and leads to a lift coefficient decrease from $C_{L}=1.01$ to $C_{L}=0.93$.

Experimental high level of fluctuations related in Figure 10 have a periodic behavior. Figures 14 (a), (b) and (c) show a frequency $f_{11}=550 \mathrm{~Hz}$ that disappears at $t=0.92 \mathrm{~s}$ corresponding to $\alpha=5.5^{\circ}$. The frequency results from a global instability in the reattachment region [34]. This leads to a flapping motion of the separated shear layer. It moves from transducers $x / c=0.9$ to $x / c=0.7$ because the LSB is moving progressively to the leading edge as the angle of incidence increases. Higher frequencies visible in Figure 14 (a) and mostly Figure 14 (d) are harmonics of $f_{11}=550 \mathrm{~Hz}$. A peak is visible after $t=0 \mathrm{~s}$ at $f_{12} \approx 1100 \mathrm{~Hz}$ for $x / \mathrm{c}=0.9$ and moves up to $x / c=0.8$ at $t=0.35 \mathrm{~s}$. Other harmonics are clearly visible at $t=0.45 \mathrm{~s}$ for $x / c=0.8$, values are $f_{12} \approx 1100 \mathrm{~Hz}, f_{13} \approx 1650 \mathrm{~Hz}$ and $f_{14} \approx 2100 \mathrm{~Hz}$. Those frequencies result from a turbulence development induced by global instability at $f_{11}=550 \mathrm{~Hz}$. Haggmark et al. (2000) [34] has found that disturbance over LSB can induce low and high frequency fluctuations.

Figure 14 (e) shows a frequency of $f_{2}=1700 \mathrm{~Hz}$ which is clearly identified as a transition shift at the leading edge. This frequency is also interpreted as a vortex shedding frequency induced by LSB at leading edge. The transducer 
located upstream at $x / c=0.2$ shows a low amplitude frequency around $500 \mathrm{~Hz}$ whereas the transducer located downstream at $x / c=0.5$ shows a broad frequency spectrum one can be the consequence of a fully turbulent flow.

The Strouhal number of vortex shedding $S_{t \theta}[14]$ is evaluated using the computed boundary layer momentum thickness $(\theta)_{\text {sep }}$ and the local upstream velocity $\left(u_{e}\right)_{\text {sep }}$ at separation and the experimental shedding frequencies. Before the transition passes near the leading edge, the LSB is located in a thick and developed boundary layer discretized into 20 layers. Computation gives mean values of $(\theta)_{\text {sep }}=10.12 \times 10^{-5} \mathrm{~m}$ and $\left(u_{e}\right)_{\text {sep }}=6.28 \mathrm{~m} / \mathrm{s}$ for $\alpha$ from $0^{\circ}$ to $5^{\circ}$. This leads to $S_{t \theta}=0.0088$ that can be compared to the $S_{t \theta}=0.00686$ found by Pauley et al (1990) in [14]. Computed boundary layer momentum thickness $(\theta)_{\text {sep }}$ and velocity $\left(u_{e}\right)_{\text {sep }}$ for $\alpha$ from $6^{\circ}$ to $12^{\circ}$ are respectively between $(\theta)_{\text {sep }}=1.0 \times 10^{-5} \mathrm{~m}$ to $1.5 \times 10^{-5} \mathrm{~m}$ and $\left(u_{e}\right)_{\mathrm{sep}}=7.135 \mathrm{~m} / \mathrm{s}$ to $8.135 \mathrm{~m} / \mathrm{s}$ at separation point. It leads to $S_{t \theta}=0.00238$ to 0.00313 .

A roughness patch has been added on the leading edge in order to trigger the turbulence. Figure 15 shows the comparison of pressure fluctuations with and without roughness for $x / c=0.8$ and $x / c=0.3$. The pressure fluctuations relied to LSB vortex shedding are not visible anymore on pressure signal with roughness. Fluctuations are week for $\alpha=0^{\circ}$ to $5^{\circ}$ at $x / c=0.8$ (trailing edge) as well as for $\alpha=5^{\circ}$ to $12^{\circ}$ at $x / c=0.3$ (leading edge). Except for those zones, pressure fluctuations are the same. This clearly shows that the high pressure fluctuations observed earlier are relied to the presence of a LSB and to the transition to turbulence. The corresponding spectrograms of the signal obtained with roughness are shown on Figures 15 (c) and 
(d) and confirm this result. Frequencies of $f=550 \mathrm{~Hz}$ and $f=1700 \mathrm{~Hz}$ have disappeared on both transducers. A low frequency around $200 \mathrm{~Hz}$ is observed and could be the consequence of the roughness patch; this has to be confirmed by future works.

\subsection{Dynamic effects of pitching velocity on local pressure and boundary layer events}

Figure 16 is a comparison between numerical and experimental local wall pressures at $x / c=0.3$ and $x / c=0.8$ for pitching velocities ranging between $\dot{\alpha}^{*}=0.618$ and $\dot{\alpha}^{*}=1.89$. The increase of transient effect when pitching velocity increase does not affects the numerical prediction and the pressure variations due to laminar to turbulent transition is well predicted by computation in all cases. In particular, the pressure inflection resulting from the moves of the transition at the leading edge ( at $\alpha=5^{\circ}$ for $\dot{\alpha}^{*}=0.18$ see the previous section) can be seen for both computation and experiments. The transition is delayed when pitching velocity increase, as a consequence the transducer located at $x / c=0.8$ Figure 16 (a) show a pressure inflection at $\alpha=6 \circ$ for $\dot{\alpha}^{*}=0.618$ whereas it is delayed at $\alpha=9 \circ$ for $\dot{\alpha}^{*}=1.89$ as shown in Figure 16 (e).

Figure 18 (a) shows the transition and the trailing edge separation and Figure 18 (b) shows the LSB length, for the considered pitching velocities. It is observed that transition is delayed when pitching velocity increases. As a consequence, trailing edge separation point is delayed which induces a higher lift coefficient before stall. Pitching velocity does not have impact on the LSB size. Few variations appear when it forms but from $\alpha=7^{\circ}$ to stall, bubble 
lengths are the same. This correlation shows that high pitching velocities delay the separation induced transition phenomenon whereas boundary layer thickness and separation length are conserved. All these points have an impact on hydrodynamic loading of the foil.

Spectrograms of the experimental wall pressure measurements are shown in Figure 17. Frequencies observed on transducers at $x / c=0.3$ and $x / c=0.8$ are constant as functions of pitching velocity and are delayed in time compared to the lowest pitching velocity. It means that the transition phenomenon is delayed as well.

\subsection{Suction side loading analysis}

Based on available experimental data, an analysis of suction side loading can be done by summing pressure coefficients on the suction side. The approximation can be written as:

$$
C_{l}^{+}(t)=\sum_{i=2}^{10} C_{p}\left(x_{i} / c, t\right) \Delta\left(x_{i} / c\right)
$$

where $C_{p}\left(\frac{x_{i}}{c}, t\right)$ is the pressure coefficient at location $\frac{x_{i}}{c}$ and $\Delta\left(\frac{x_{i}}{c}\right)$ is the non dimensional distance between two consecutive transducers. The procedure is applied to numerical data for comparison.

Figure 19 shows the results obtained for the 4 pitching velocities. As shown, there is a good agreement between measurements and computations. The difference is very weak at the beginning of the pitching rotation and the inflection which appears at $5^{\circ}$ for the lowest pitching velocity is accurately predicted by the transition model. It is delayed when pitching velocity increases and disappears even completely for the highest pitching velocity for 
both experimental and numerical approaches. High amplitude fluctuations at low frequency induced by leading edge vortex shedding are over predicted by computations but the phenomenon starts at an angle of incidence very close to measurement. This over prediction can be explained by $3 \mathrm{D}$ effects which develop along the suction side due to large structure of vortex shedding which are not fully considered in the $2 \mathrm{D}$ computation. The return to $0^{\circ}$ shows hysteresis induced by a delay in the reattachment. Again, computations agree well to measurements even if the model tends to over predict the loading when the pitching velocity increases. Then it allows the authors to analyze the influence of pitching velocity on hydrodynamic coefficients obtained by computations.

\subsection{Dynamic effects of pitching velocity on hydrodynamic coefficients}

Figure 20 shows the numerical lift coefficient evolution as a function of the angle of incidence during pitching motion. This one is obtained by the integration of pressure over the whole foil surface. Transition model appears to have a significant impact on lift when the pitching velocity is weak. An inflection is followed by a slope modification at $5^{\circ}$ for $\dot{\alpha}^{*}=0.18$ and $7^{\circ}$ for $\dot{\alpha}^{*}=1.05$ which tends to disappear for $\dot{\alpha}^{*}=1.89$. So the highest pitching velocities tend to delay the transition of the boundary layer as well as it deletes the effect on hydrodynamic loading. This can be due to the diffusive time of the viscosity which becomes too high as compared to the pitching velocity. Then, we see that lift amplitude before stall is higher for high velocities. Stall appears at $13.3^{\circ}$ for the lowest velocity and is delayed at $14.4^{\circ}$ for the highest velocity. It is shown that high lift fluctuations induced by leading edge vortex shedding appear for all pitching velocities, about three 
times for the lowest pitching velocity and only one time for the other ones. The flow reattachment is also delayed with high pitching velocities which induce an hysteresis effect. As a consequence, lift evolution is symmetric for $\dot{\alpha}^{*}=0.18$ where the reattachment is located for $C_{L}=1.33$ whereas $C_{L}=0.16$ for $\dot{\alpha}^{*}=1.89$.

Figure 21 shows the drag coefficient (a) and the moment coefficient (b) versus the angle of incidence. Pitching velocity has some influence before the transition passes at the leading edge for both moment and drag coefficients. For the case $\dot{\alpha}^{*}=0.18$ and $\alpha=5^{\circ}, C_{m}=0.087$ and $C_{d}=0.0124$ whereas

higher values are found for $\dot{\alpha}^{*}=1.89, C_{m}=0.107$ and $C_{d}=0.0325$. It has been shown that the boundary layer is laminar from $\alpha=0^{\circ}$ to $5^{\circ}$, so the increase can be attributed to inertia effects. Then, for the lower pitching velocity, the transition induces a slope modification of the drag curve due to the increase of wall shear stress because the boundary layer becomes turbulent. It also affects strongly the moment coefficient which decreases by a half. Again, the highest pitching velocity shows a disappearance of the transition effects. Drag coefficient is almost linear whereas a small inflection is found on the moment coefficient curve.

\section{CONCLUSION}

The spatio-temporal evolution of the wall pressure field on the suction side of a NACA66 hydrofoil arising from a transient pitching motion at a $R e=0.75 \times 10^{6}$ has been carried out for both experimental and numerical approaches. Computations are performed using RANS equations including a transition model. Four pitching velocities have been studied from a low one 
$\dot{\alpha}^{*}=0.18$ to a high one $\dot{\alpha}^{*}=1.89$ for an angle of incidence varying between 0 and $15^{\circ}$. The repartition of pressure transducers along the chord on the suction side of the hydrofoil at reduced coordinates located between $x / c=0.10$ and $x / c=0.90$ with a step of $0.10 \mathrm{c}$ allowed us both global and local analysis. Local wall pressure coefficients allow to qualify the boundary layer transition. It is characterized by a pressure inflection during the foil rotation and periodic pressure fluctuations induced by vortex shedding of a laminar separation bubble. The LSB occurs near the trailing edge for low angles of incidence and passes suddenly close to the leading edge for larger angles of incidence. This is confirmed by the experiments conducted with a roughness patch added at the leading edge which suppresses the characteristic periodic pressure fluctuations. LSB and transition are accurately predicted numerically except for periodic wall pressure fluctuations which are not captured by the RANS code. The influence of pitching velocity was studied. It was observed that the increase of pitching velocity delays the boundary layer transition from $5^{\circ}$ for the lowest pitching velocity to $8^{\circ}$ for the highest pitching velocity. On the other hand, computed leading edge LSB length and experimental frequencies resulting from periodic pressure fluctuations are constant versus pitching velocity.

Suction side loading shows a good agreement between measurements and computations. The impact of transition for various pitching velocities was observed on lift coefficient. For a low pitching velocity, the transition induced a significant lift coefficient inflection at $\alpha=5^{\circ}$. For higher pitching velocities the lift inflection is reduced and totally suppressed for the highest pitching velocity. In the latter case, the hydrofoil loading is strongly modified leading 
to higher lift values at stall together with a strong hysteresis effect during the back motion from $\alpha=15^{\circ}$ to $\alpha=0^{\circ}$.

\section{acknowledgment}

The authors gratefully acknowledge the technical staff of IRENav for its contribution to the experimental set up.

\section{References}

[1] G. S. Triantafyllou, M. S. Triantafyllou, M. Grosenbaugh, Optimal thrust development in oscillating foils with application to fish propulsion., Journal of Fluids and Structures 7 (2) (1993) 205-224.

[2] G. R. Srinivasan, J. A. Ekaterinaris, W. J. McCroskey, Evaluation of Turbulence Models for Unsteady Flows of an Oscillating Airfoil, Pergamon; National Aeronautics and Space Administration; National Technical Information Service, distributor, 1995.

[3] G. N. Barakos, Unsteady separated flows over manoeuvring lifting surfaces, Philosophical Transactions: Mathematical, Physical and Engineering Sciences 358 (1777) (2000) 3279-3291.

[4] H. Hamdani, M. Sun, Aerodynamic forces and flow structures of an airfoil in some unsteady motions at small Reynolds number, Acta Mechanica 145 (1) (2000) 173-187.

[5] T. Lee, P. Gerontakos, Investigation of flow over an oscillating airfoil, Journal of Fluid Mechanics 512 (2004) 313-341. 
[6] A. Shelton, J. Abras, B. Hathaway, M. Sanchez-Rocha, M. J. Smith, S. Menon, An Investigation of the Numerical Prediction of Static and Dynamic Stall, Proceedings of the 61" American Helicopter Society Annual Forum, Grapevine 10 (10) (2005) 6.

[7] G. Studer, Experimental and numerical study of laminar-turbulent transition in unsteady flow, Ph.D. thesis, Ecole nationale supérieure de l'aéronautique et de l'espace (2005).

[8] F. Richez, I. Mary, V. Gleize, C. Basdevant, Near stall simulation of the flow around an airfoil using zonal RANS/LES coupling method, Computers and Fluids 37 (7) (2008) 857-866.

[9] M. J. Smith, T. C. Wong, M. Potsdam, J. Baeder, S. Phanse, Evaluation of CFD to Determine Two-Dimensional Airfoil Characteristics for Rotorcraft Applications, in: American Helicopter Society 60th Annual Forum, Baltimore, MD, June 7-10, 2004.

[10] I. Tani, Low-speed flows involving bubble separations, Prog. Aerosp. Sci 5 (1964) 70-103.

[11] M. Gaster, The Structure and Behaviour of Laminar Separation Bubbles, HMS O, 1969.

[12] M. Alam, N. D. Sandham, Direct numerical simulation of shortlaminar separation bubbles with turbulent reattachment, Journal of Fluid Mechanics 410 (2000) 1-28.

[13] A. E. Alving, H. H. Fernholz, Turbulence measurements around a mild 
separation bubble and downstream of reattachment, Journal of Fluid Mechanics Digital Archive 322 (2006) 297-328.

[14] L. L. Pauley, P. Moin, W. C. Reynolds, The structure of two-dimensional separation, Journal of Fluid Mechanics 220 (1990) 397-411.

[15] Z. Yang, P. R. Voke, Large-eddy simulation of boundary-layer separation and transition at a change of surface curvature, Journal of Fluid Mechanics 439 (2001) 305-333.

[16] M. Marquillie, U. Ehrenstein, On the onset of nonlinear oscillations in a separating boundary-layer flow, Journal of Fluid Mechanics 490 (2003) $169-188$.

[17] U. Rist, U. Maucher, Investigations of time-growing instabilities in laminar separation bubbles, European Journal of Mechanics/B Fluids 21 (5) (2002) 495-509.

[18] L. E. Jones, R. D. Sandberg, N. D. Sandham, Direct numerical simulations of forced and unforced separation bubbles on an airfoil at incidence, Journal of fluid mechanics 602 (2008) 175.

[19] J. B. Leroux, O. Coutier-Delgosha, J. A. Astolfi, A joint experimental and numerical study of mechanisms associated to instability of partial cavitation on two-dimensional hydrofoil, Physics of Fluids 17 (2005) 052101.

[20] N. E. Huang, Z. Shen, S. R. Long, H. H. Wu, M. C.and Shin, Q. Zheng, N. C. Yen, C. C. Tung, L. H. H, The Empirical Mode Decomposition 
and the Hilbert spectrum for nonlinear and non-stationary time series analysis, Proc. Royal Soc. London A vol. 454 (1998) pp. 903-995.

[21] S. Benramdane, J. C. Cexus, J. A. Astolfi, A. O. Boudraa, Timefrequency analysis of pressure fluctuations on a hydrofoil undergoing a transient pitching motion using huang-hilbert and huang-teager transforms, in: ASME PVP-2007/CREEP-8 confernce. July 22-26. San Antonio, Texas, USA., 2007.

[22] A. Ducoin, S. Benramdane, J. A. Astolfi, F. Deniset, Etude exprimentale et numérique du champ de pression pariétale sur corps portant en mouvement forcé, in: 18eme Congres Français de Mécanique, 2007.

[23] S. Benramdane, J. A. Astolfi, J. C. Cexus, A. Boudraa, Etude expérimentale du champ de pression pariétale sur un hydrofoil en mouvement forcé transitoire, in: in Proceedings des 11emes journes de l'hydrodynamique, 3-5 avril, Brest, France, 2005.

[24] T. BARTH, D. JESPERSEN, The design and application of upwind schemes on unstructured meshes, in: AIAA, Aerospace Sciences Meeting, 27 th, Reno, NV, 1989.

[25] F. R. Menter, Improved Two-Equation k-Turbulence Models for Aerodynamic Flows, NASA Technical Memorandum 103975 (1993) 34.

[26] F. R. Menter, M. Kuntz, R. Langtry, Ten Years of Industrial Experience with the SST Turbulence Model, Turbulence, Heat and Mass Transfer 4 (2003) 625-632. 
[27] W. Haase, B. Aupoix, U. Bunge, D. Schwamborn, FLOMANIA-A European Initiative on Flow Physics Modelling, Berlin: Springer, 2006. ISBN 3-540-28786-8.

[28] B. J. Abu-Ghannam, R. Shaw, Natural transition of boundary layersThe effects of turbulence, pressure gradient, and flow history, Journal of Mechanical Engineering Science 22 (1980) 213-228.

[29] J. Steelant, E. Dick, Modelling of bypass transition with conditionned intermittency transport equation, International journal for numerical methods in fluids 23 (1996) 193-220.

[30] F. R. Menter, R. Langtry, S. Völker, Transition Modelling for General Purpose CFD Codes, Flow, Turbulence and Combustion 77 (1) (2006) 277-303.

[31] N. Maman, C. Farhat, Matching fluid and structure meshes for aeroelastic computations: A parallel approach, Computers and Structures 54 (4) (1995) 779-785.

[32] C. Farhat, M. Lesoinne, P. Le Tallec, Load and motion transfer algorithms for fluid/structure interaction problems with non-matching discrete interfaces: Momentum and energy conservation, optimal discretization and application to aeroelasticity, Computer Methods in Applied Mechanics and Engineering 157 (1-2) (1998) 95-114.

[33] T. E. Tezduyar, S. Sathe, R. Keedy, K. Stein, Space-time finite element techniques for computation of fluid-structure interactions, Com- 
puter Methods in Applied Mechanics and Engineering 195 (17-18) (2006) $2002-2027$.

[34] C. P. Haggmark, A. A. Bakchinov, P. H. Alfredsson, Experiments on a two-dimensional laminar separation bubble, Philosophical Transactions: Mathematical, Physical \& Engineering Sciences 358 (1777) (2000) 31933205 . 


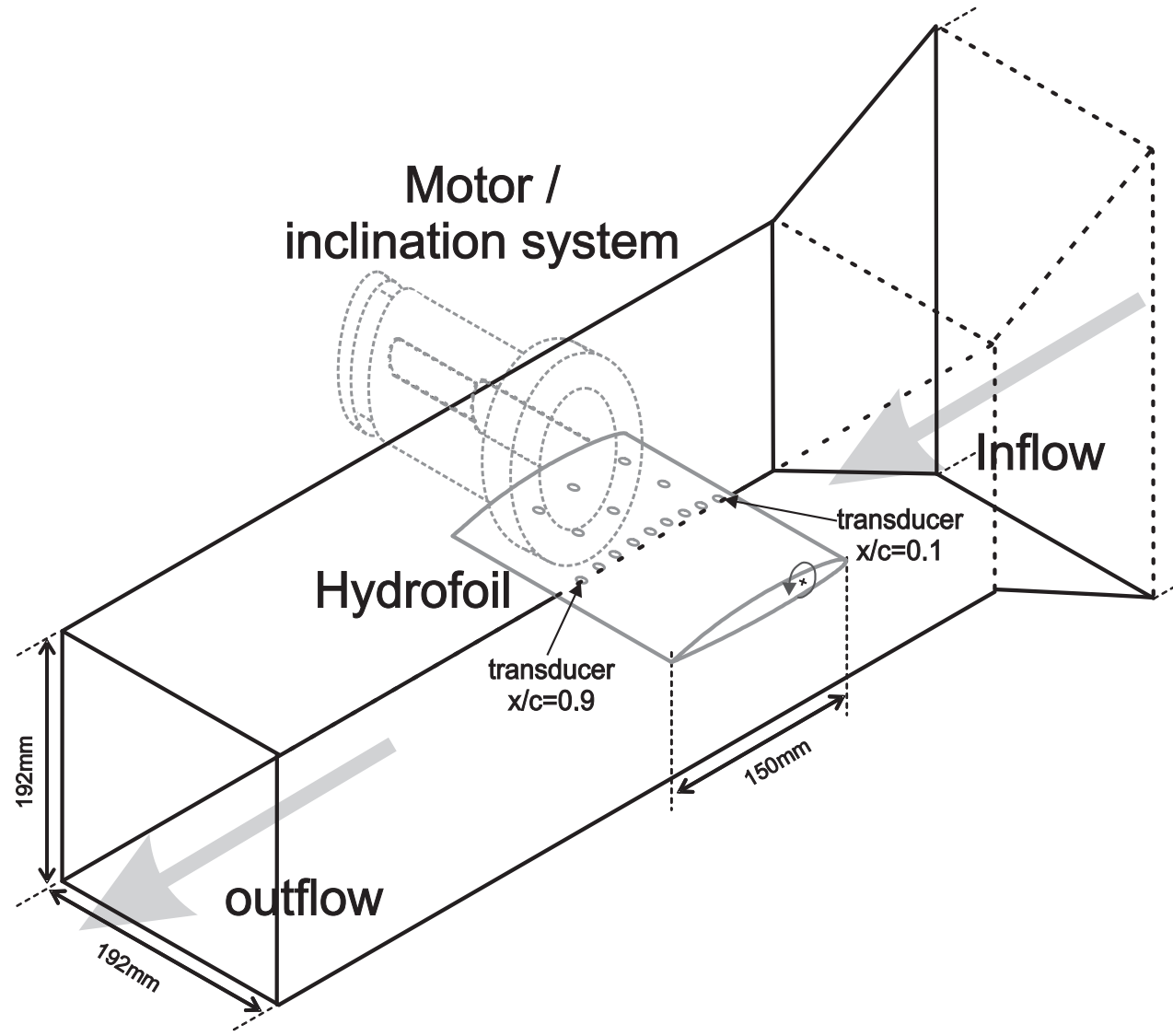

Figure 1: Hydrofoil instrumentation and tunnel test section 

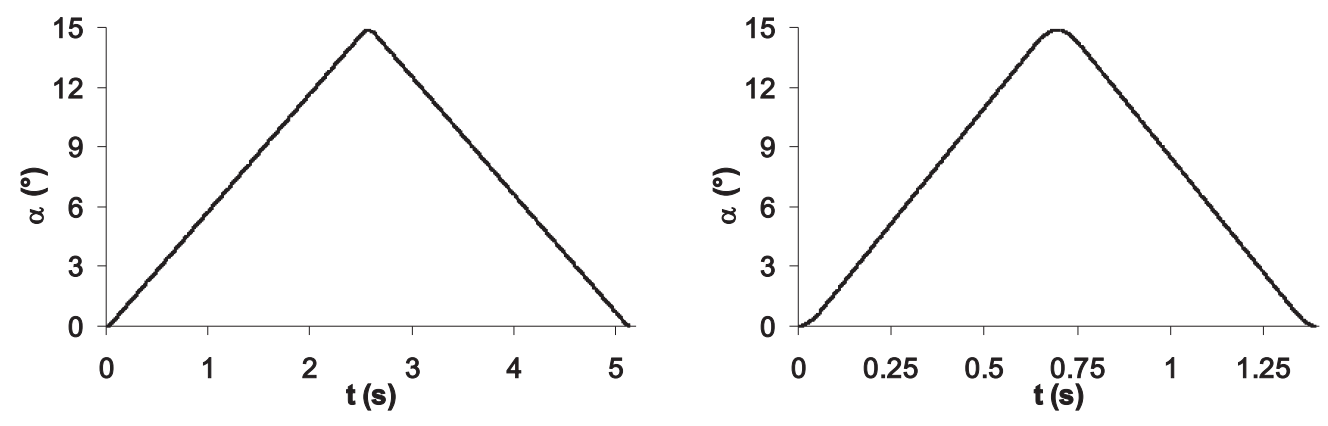

$$
\dot{\alpha}=6^{\circ} / s, \dot{\alpha}^{*}=0.18
$$$$
\dot{\alpha}=20.6^{\circ} / s, \dot{\alpha}^{*}=0.618
$$
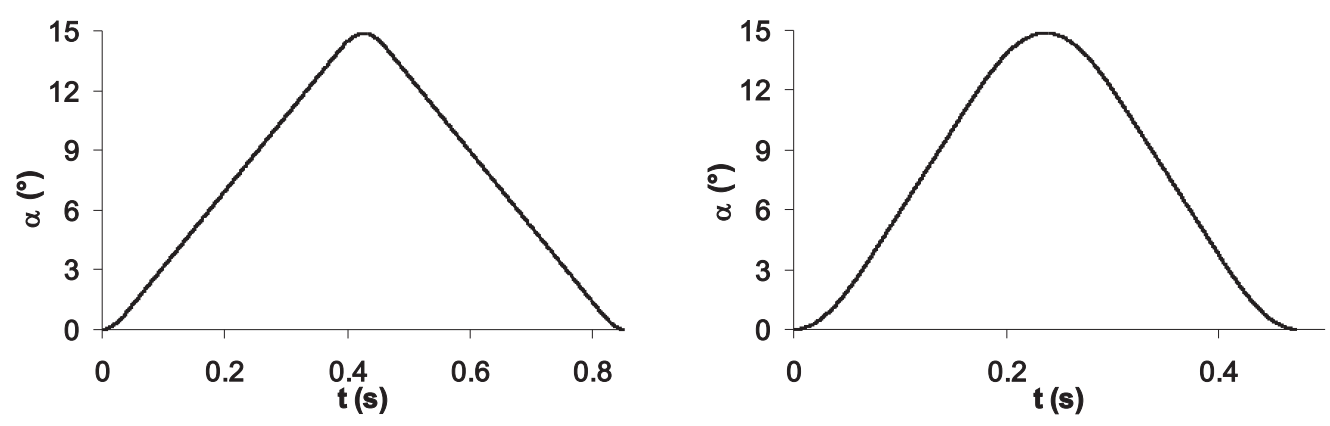

$$
\dot{\alpha}=35^{\circ} / s, \dot{\alpha}^{*}=1.05
$$

$$
\dot{\alpha}=63^{\circ} / s, \dot{\alpha}^{*}=1.89
$$

Figure 2: Measurements and computations, angle of incidence versus time for 4 pitching velocities 


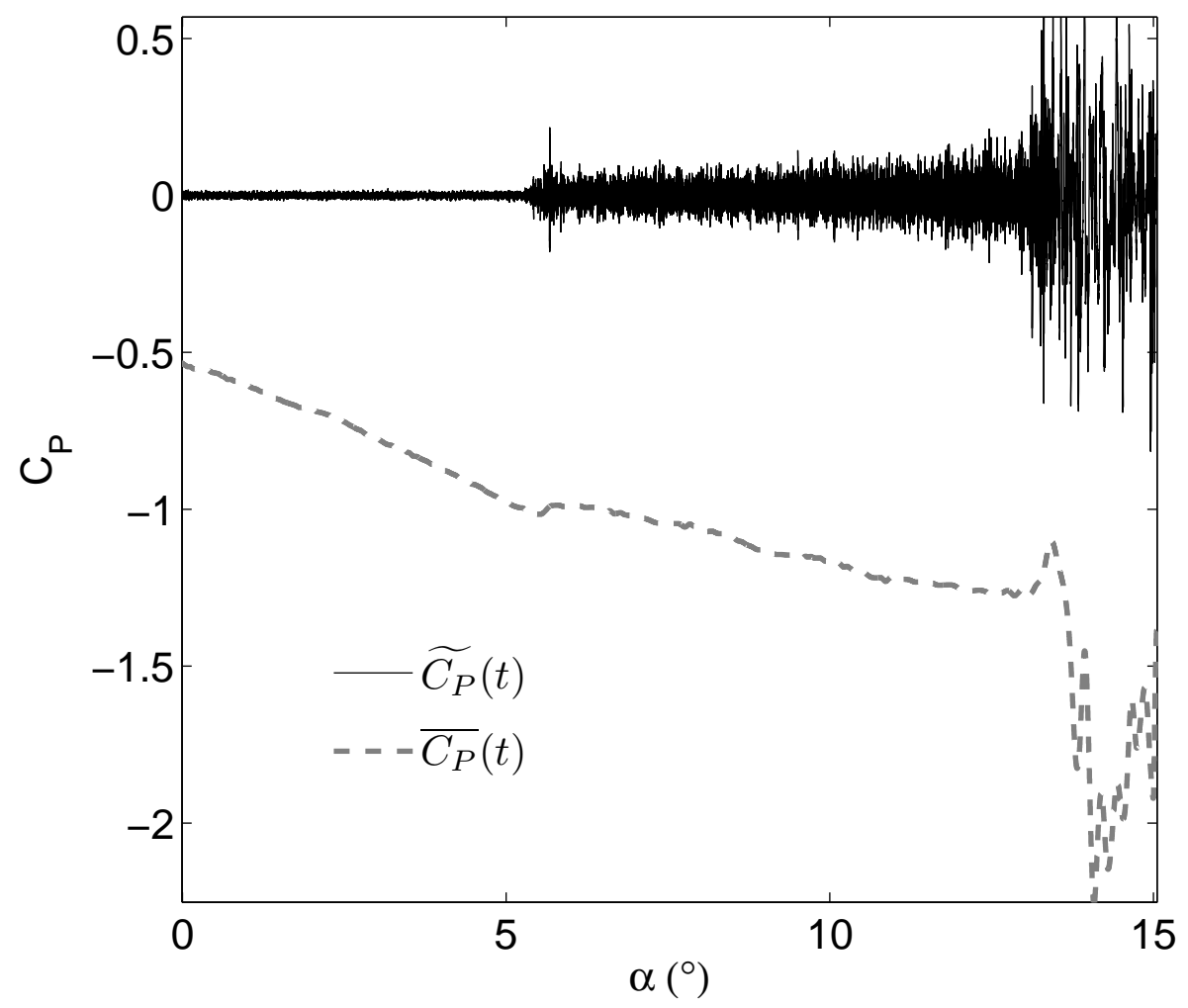

Figure 3: Measurement, reconstructed pressure signal by EMD method, $\overline{C_{P}}(t)$ (Eq. 1) and $\widetilde{C_{P}}(t)$ (Eq. 2), $\dot{\alpha}^{*}=0.18$ 


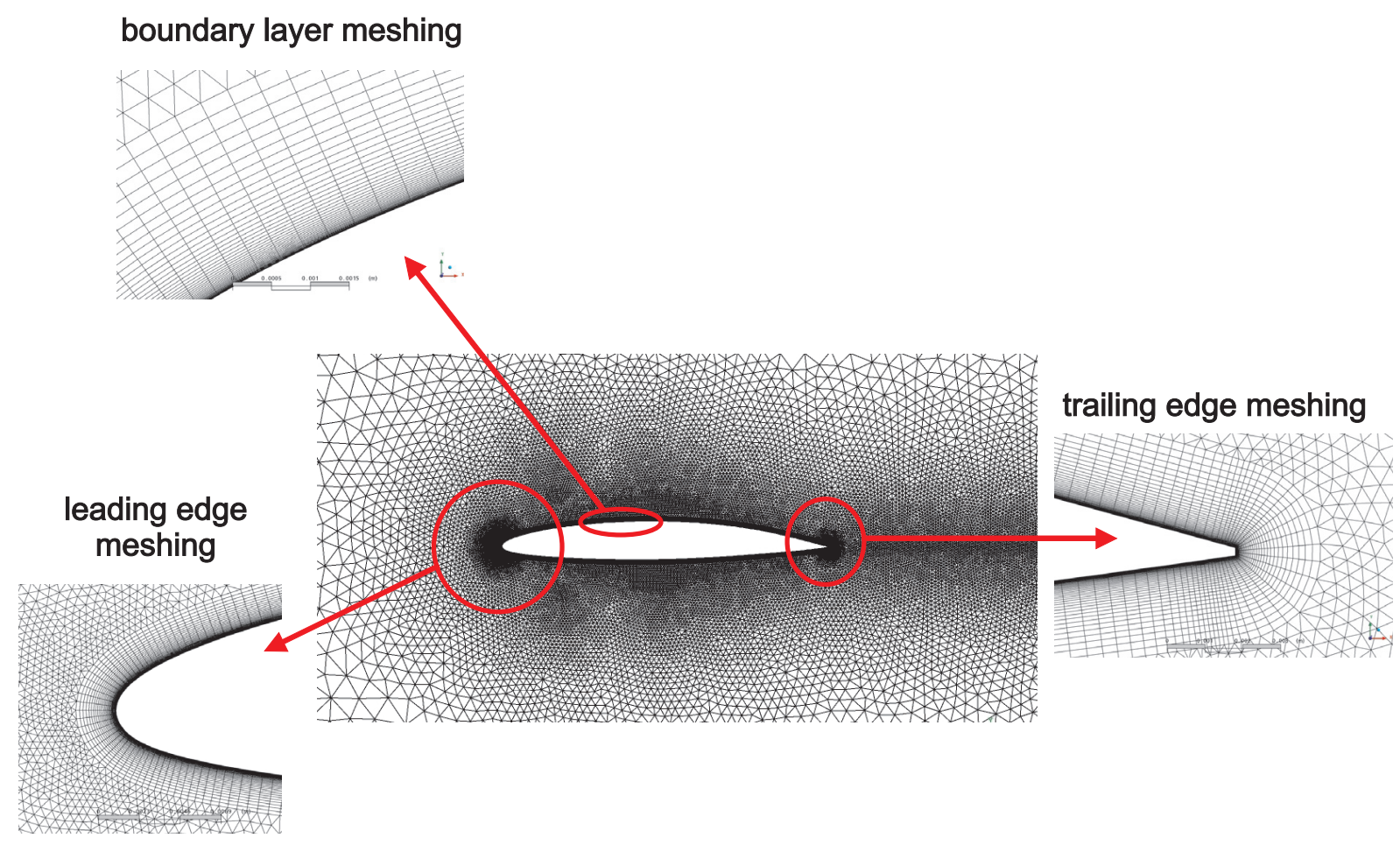

Figure 4: Hydrofoil mesh 


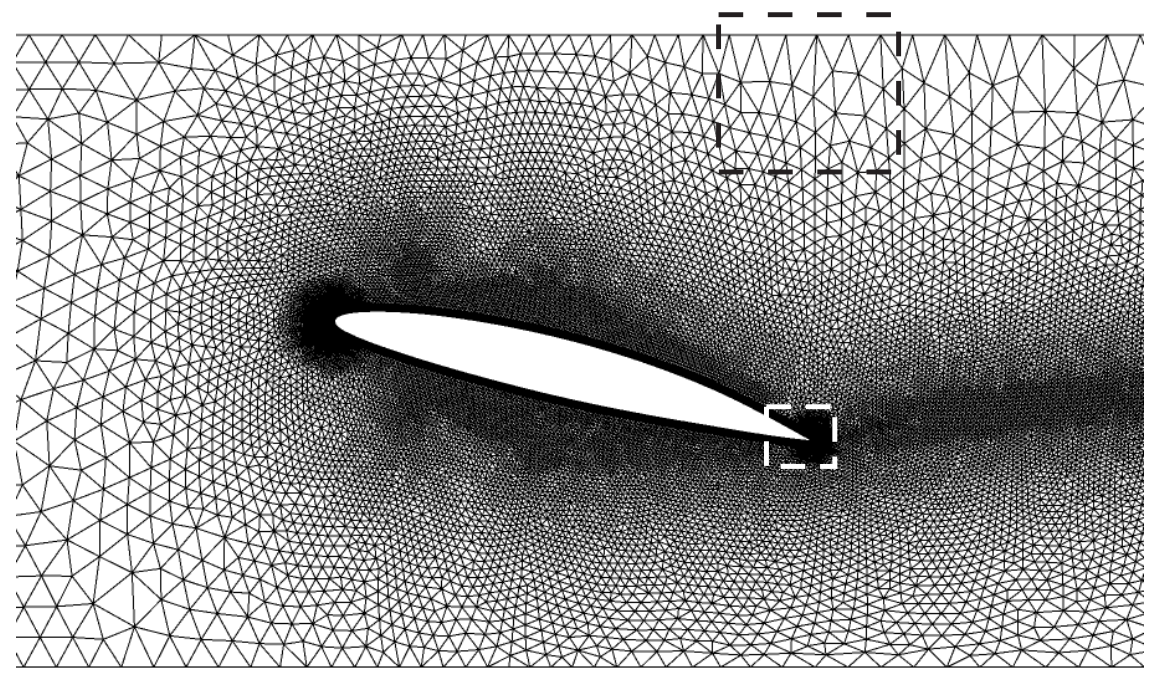

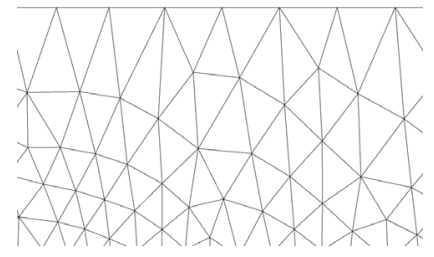

Zoom near the top wall

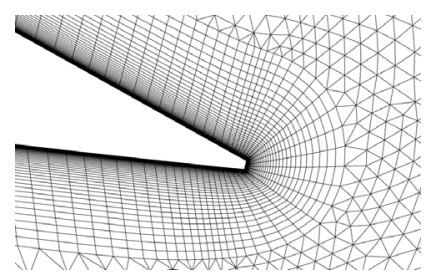

zoom near the trailing edge

Figure 5: Hydrofoil mesh after deformation, $\alpha=15^{\circ}$ 


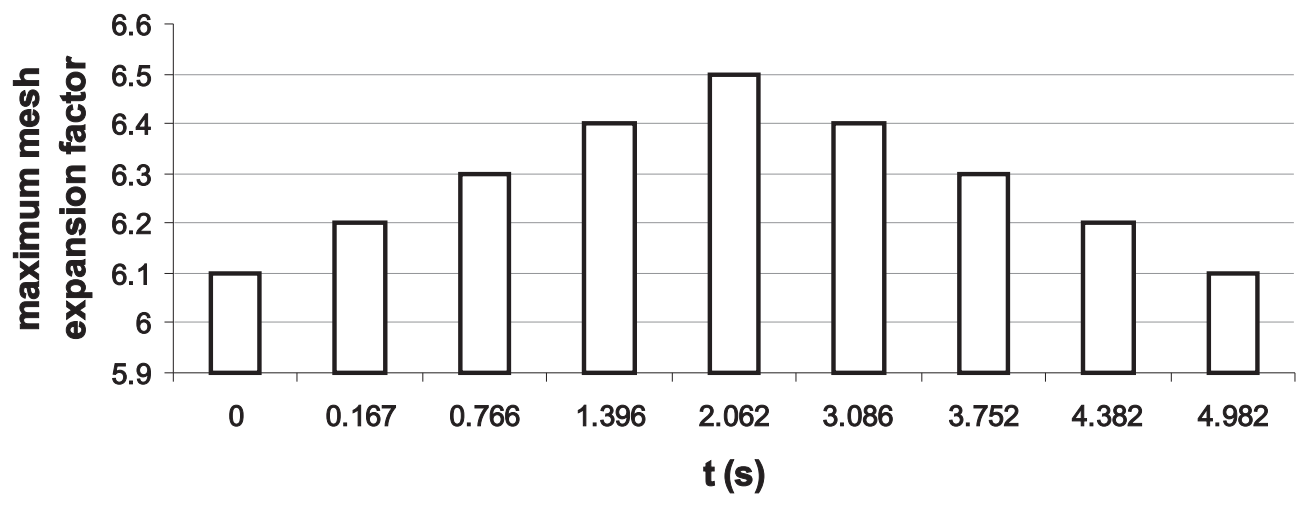

Figure 6: Mesh expansion factor versus time 


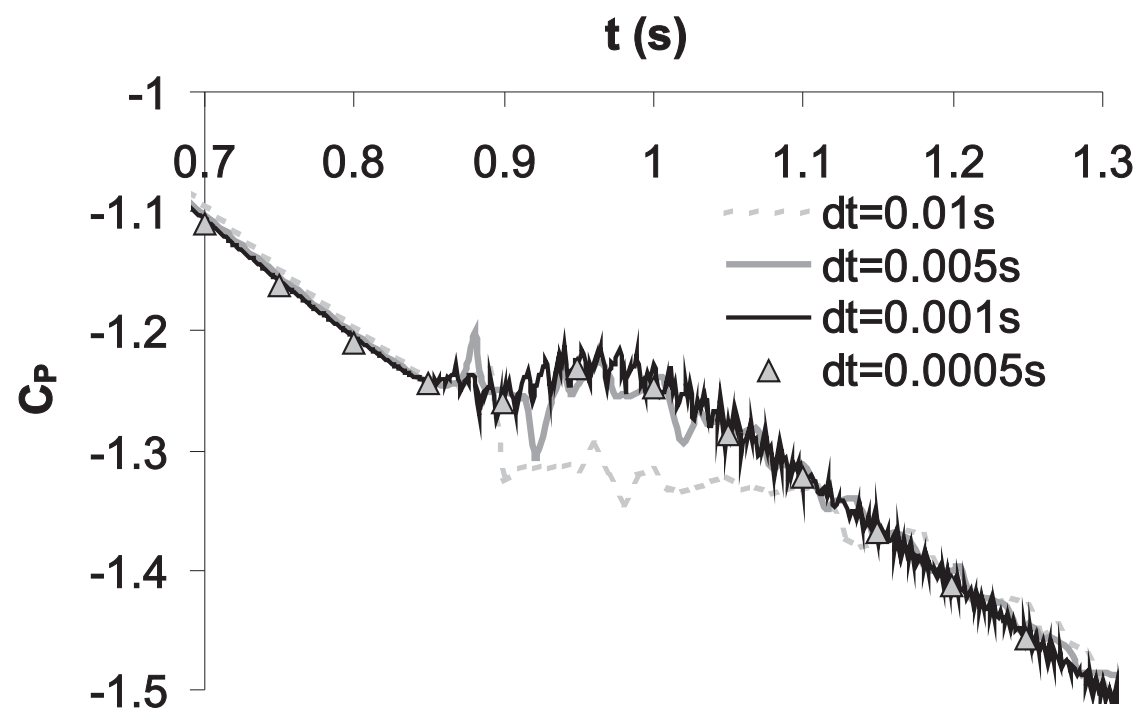

Figure 7: Computations, pressure coefficient versus time for various time steps during transition, $\dot{\alpha}^{*}=0.18, R e=750000$ 


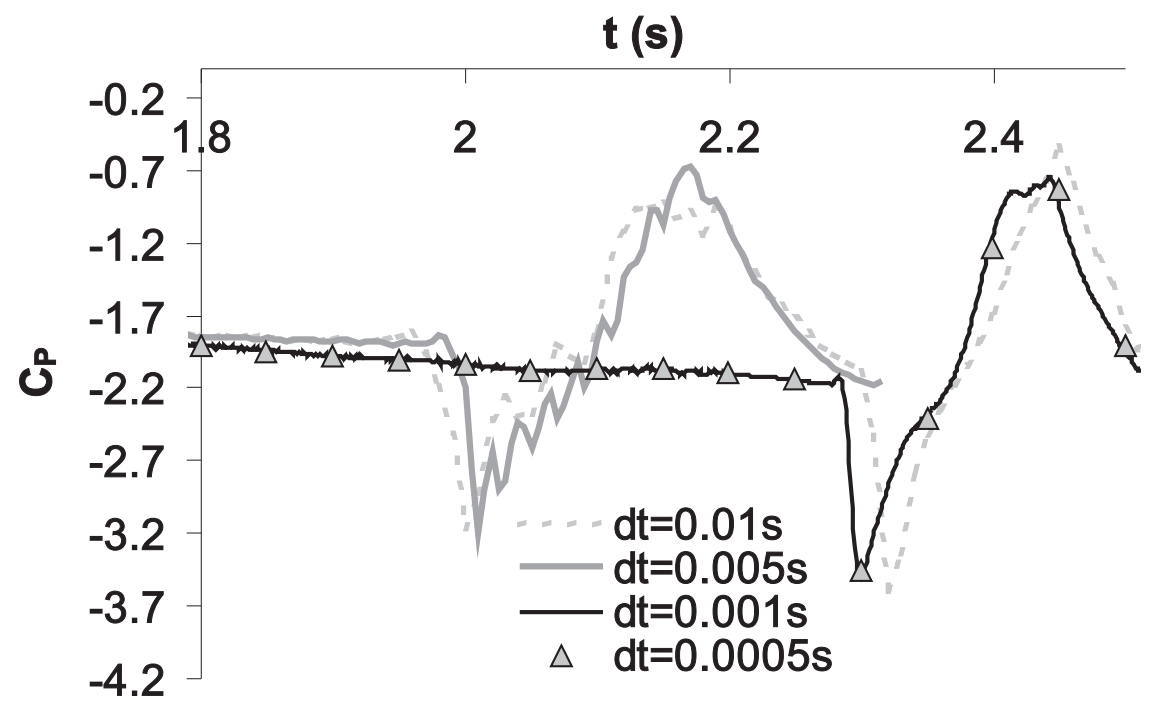

Figure 8: Computations, pressure coefficient versus time for various time steps in leading edge separation zone, $\dot{\alpha}^{*}=0.18, R e=750000$ 


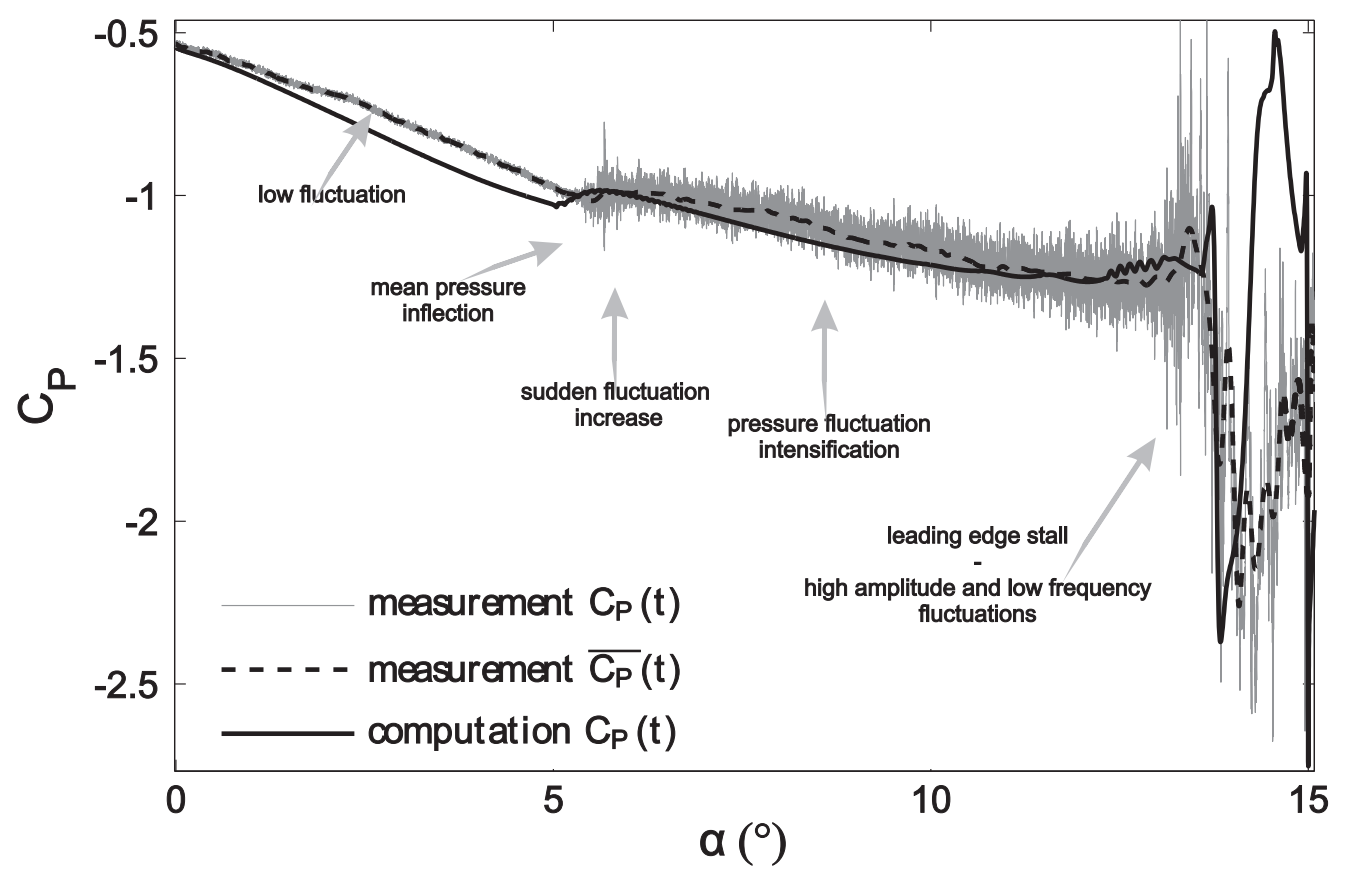

(a) $\alpha=0^{\circ}$ to $15^{\circ}$

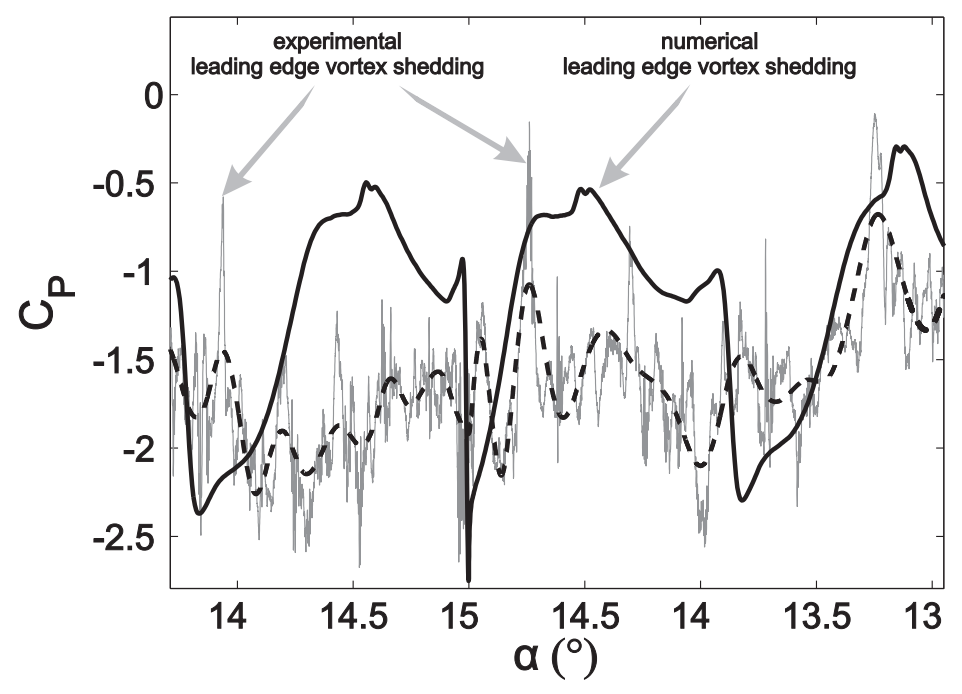

(b) $\alpha=14^{\circ}$ to $12^{\circ}$ (back step)

Figure 9: Experimental and numerical pressure coefficient as function of the angle of incidence at $x / c=0.3$ during the transient motion, $\dot{\alpha}^{*}=0.18, R e=750000$ 


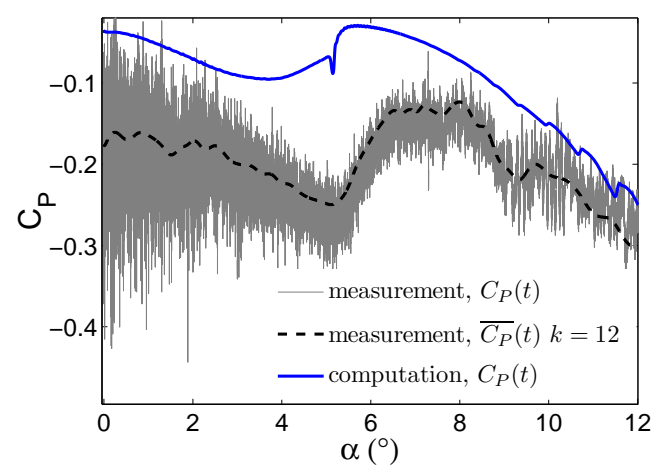

(a) $x / c=0.9$

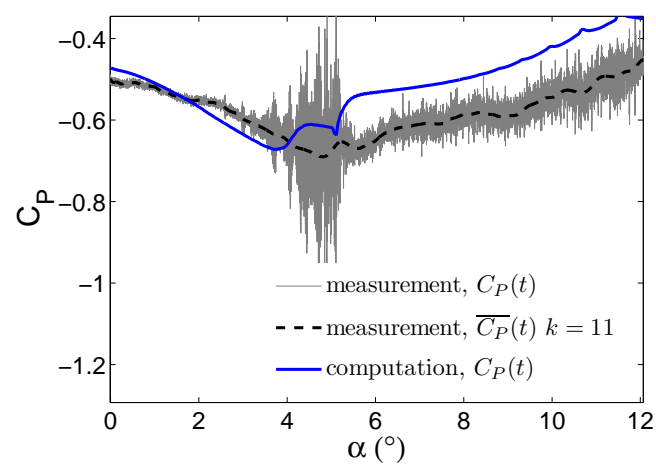

(c) $x / c=0.7$

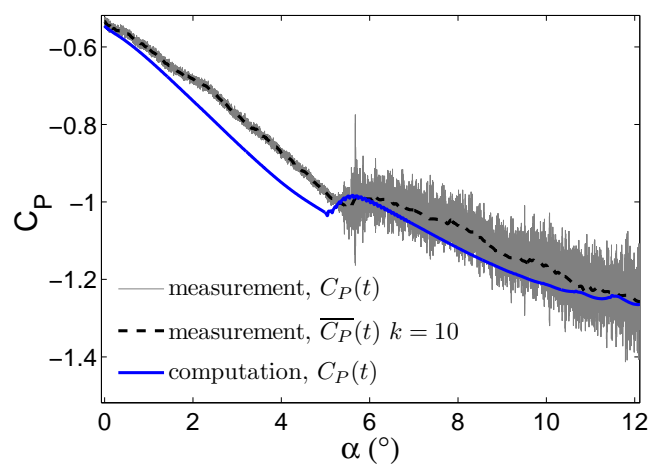

(e) $x / c=0.3$

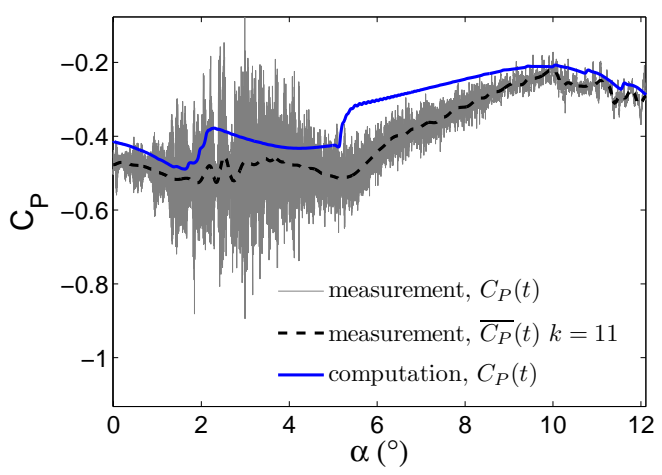

(b) $x / c=0.8$

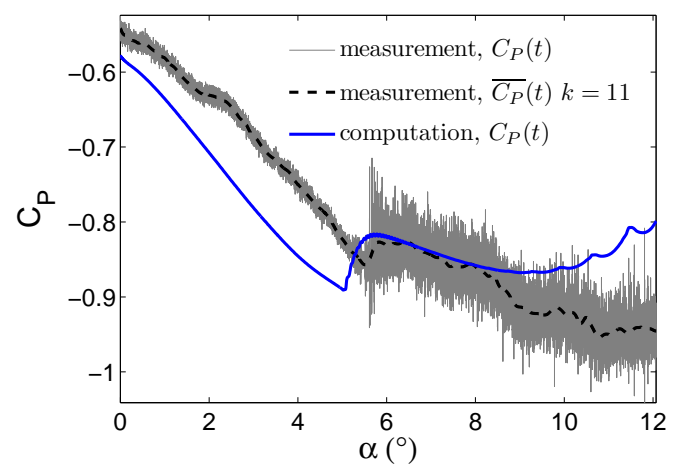

(d) $x / c=0.5$

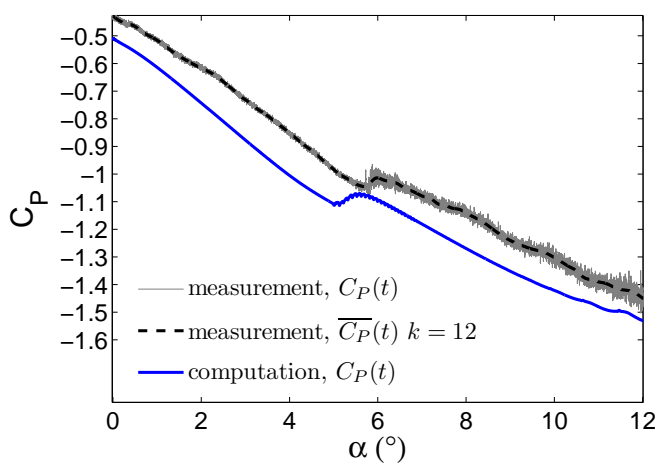

(f) $x / c=0.2$

Figure 10: Experimental and numerical pressure coefficient versus angle of incidence during the hydrofoil rotation at $\dot{\alpha}^{*}=0.18$ for various transducers along the suction side, $\alpha=0^{\circ}$ to $12^{\circ}$ before stall. The dotted lines are the low frequency EMD pressure signals $\overline{C_{P}}(t)$ (Eq. 1), $R e=750000$ 


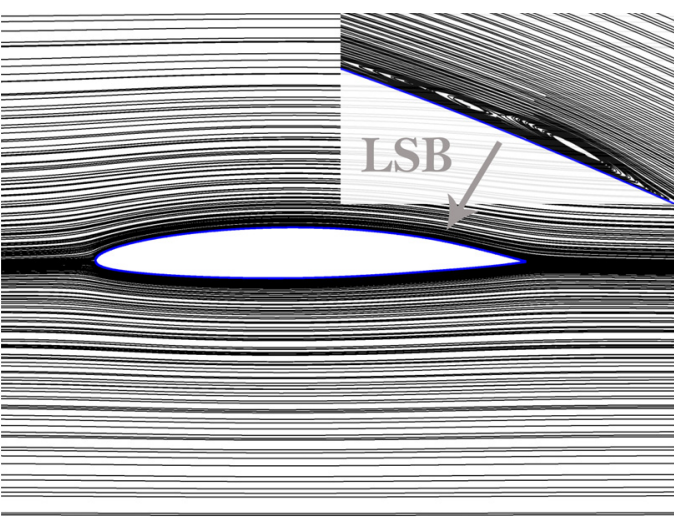

$0^{\circ}$

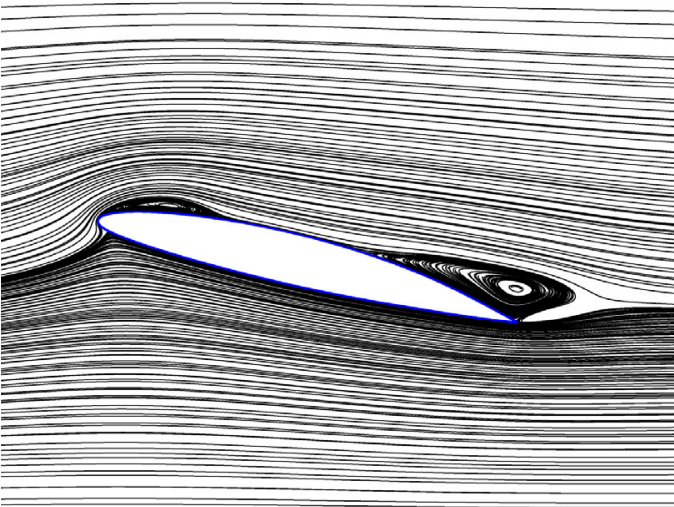

$13.6^{\circ}$

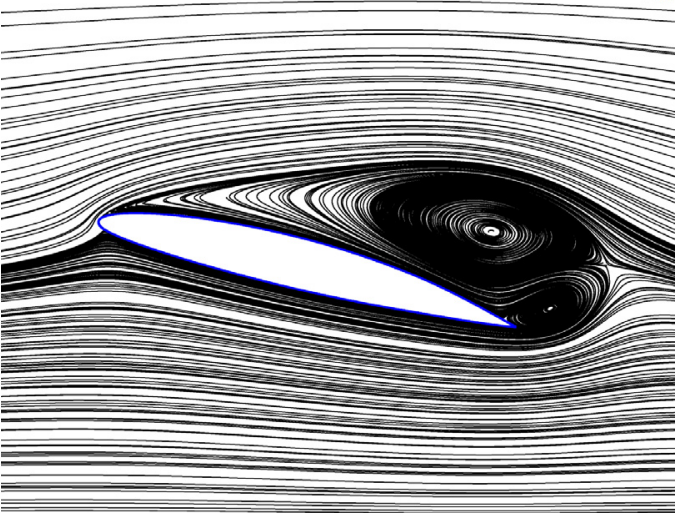

$14.2^{\circ}$

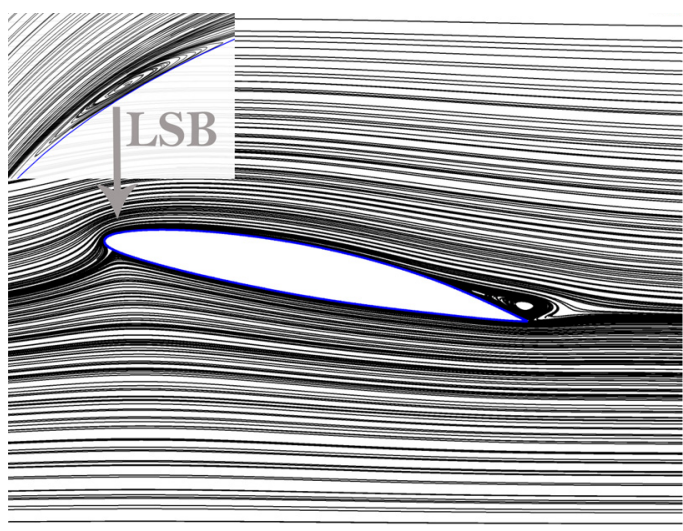

$11^{\circ}$

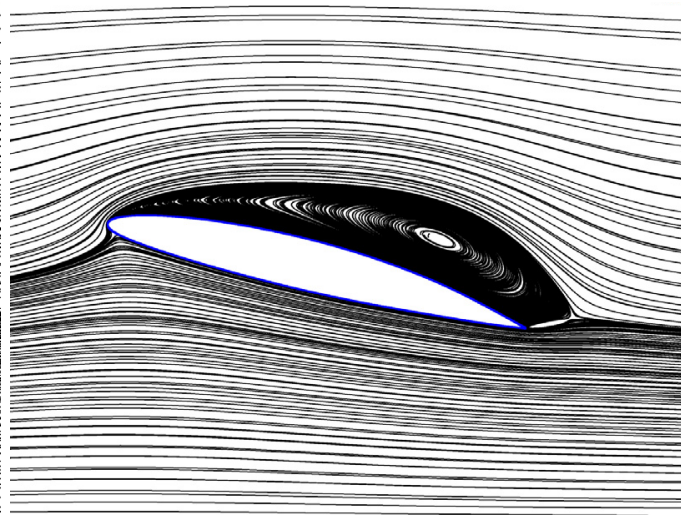

\section{$13.9^{\circ}$}

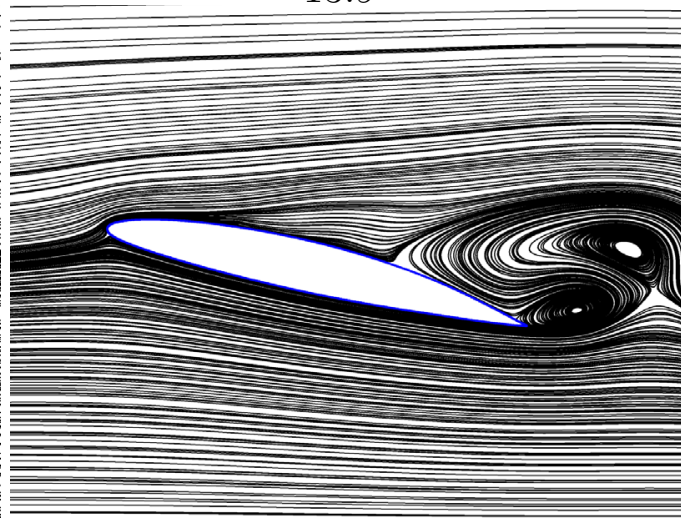

$14.5^{\circ}$

Figure 11: Computation, flow visualizations, $\dot{\alpha}^{*}=0.18$, including Laminar Separation Bubble (LSB), Re $=750000$ 


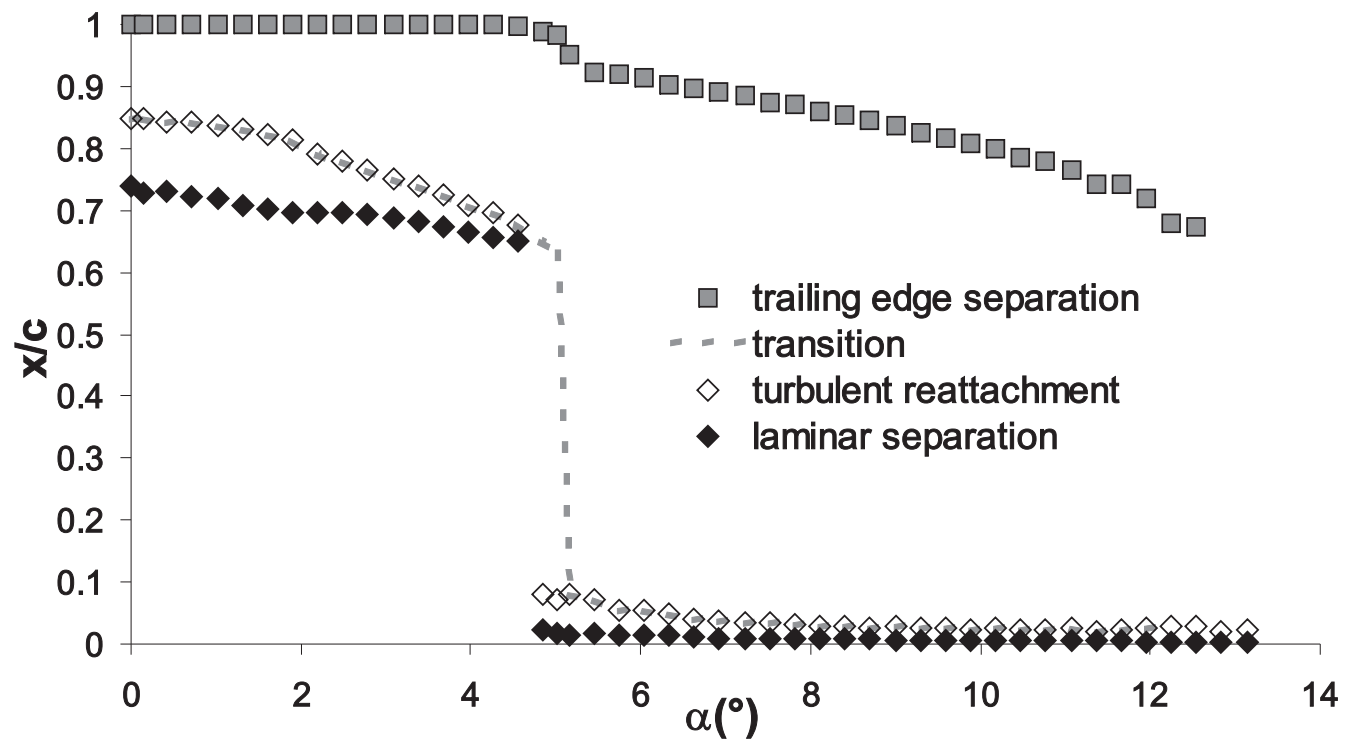

Figure 12: Computation, location of separations, reattachment and transition points on the suction side as functions of the angle of incidence during the hydrofoil rotation, $\alpha=0^{\circ}$ to $13.4^{\circ}, R e=750000, \dot{\alpha}^{*}=0.18$ 


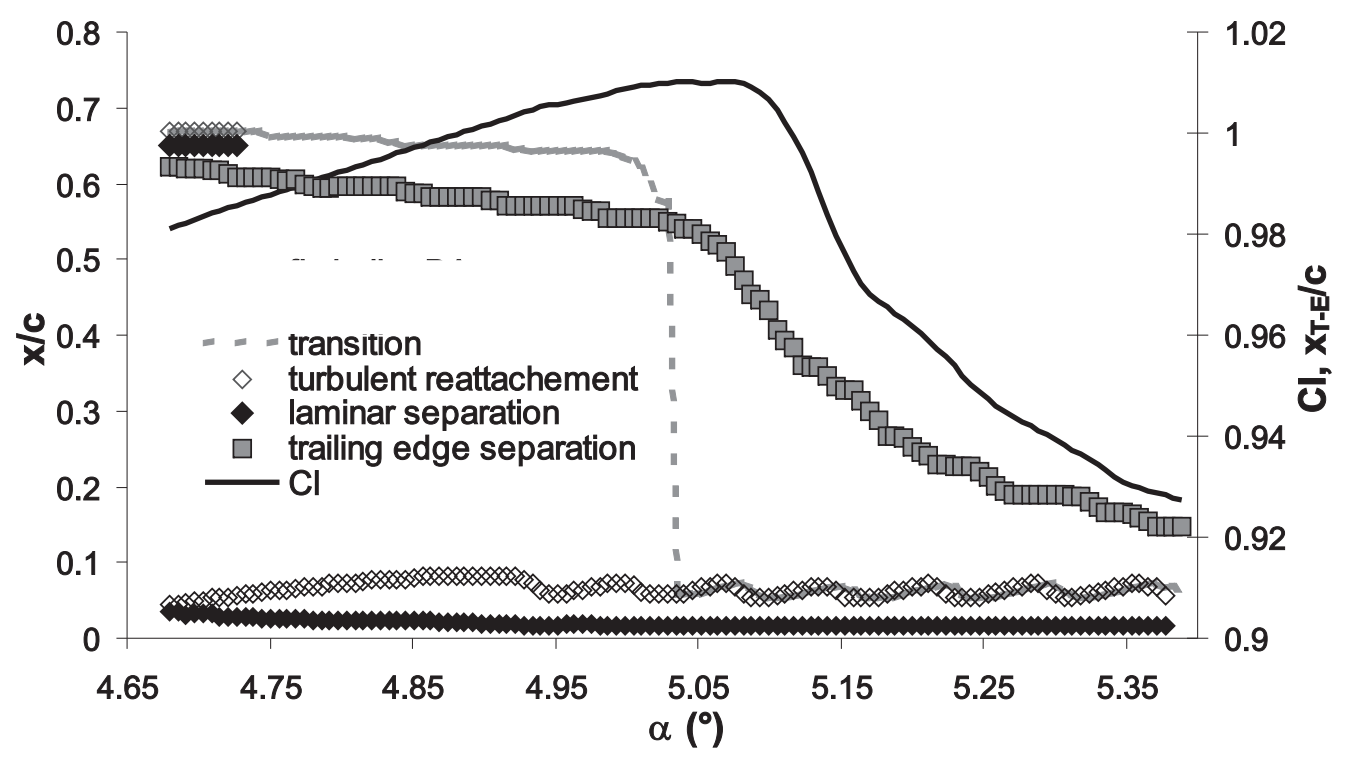

Figure 13: Computation,, location of separations, reattachment and transition points on the suction side as functions of the angle of incidence during the hydrofoil rotation, $\alpha=4.65^{\circ}$ to $5.4^{\circ}, R e=750000, \dot{\alpha}^{*}=0.18$ 


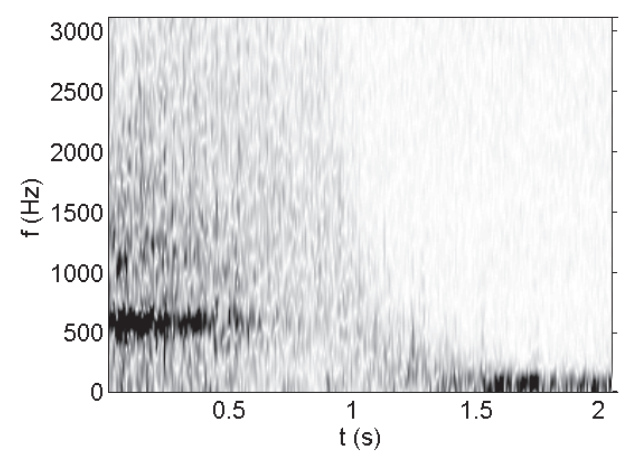

(a) $x / c=0.9, k=12$

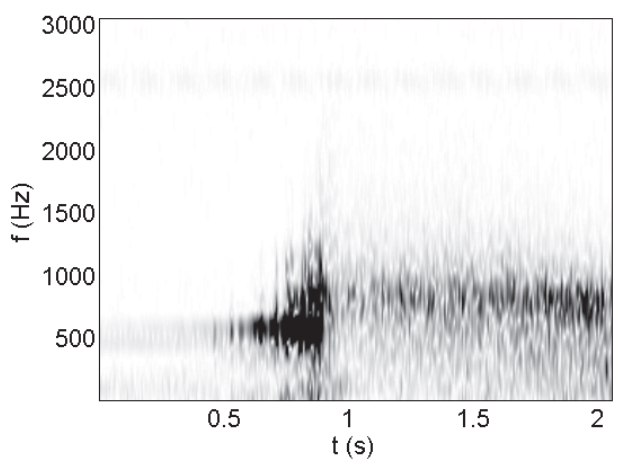

(c) $x / c=0.7, k=11$

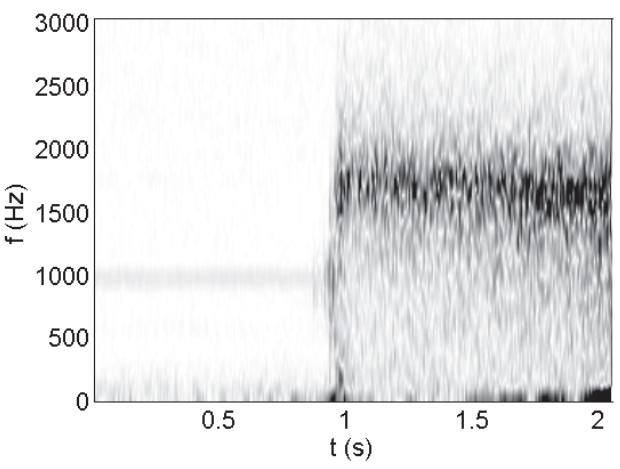

(e) $x / c=0.3, k=10$

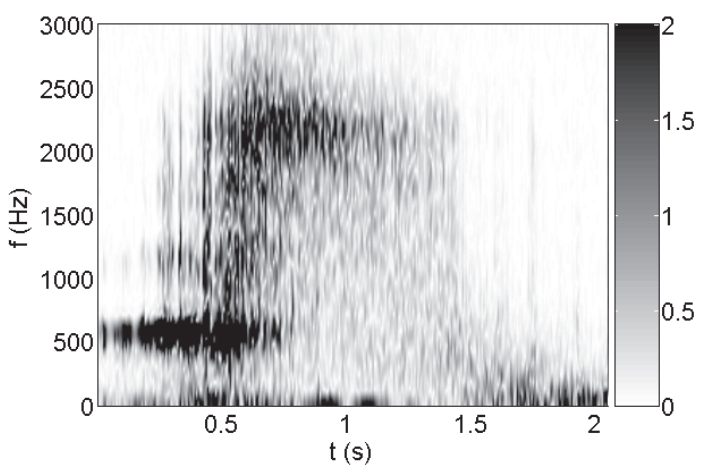

(b) $x / c=0.8, k=11$

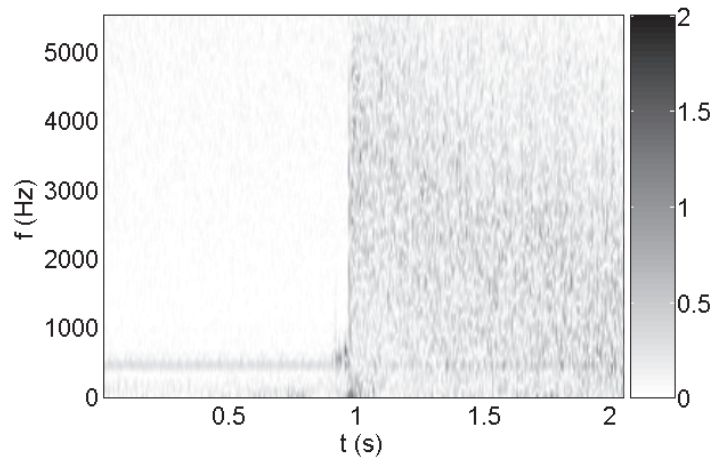

(d) $x / c=0.5, k=11$

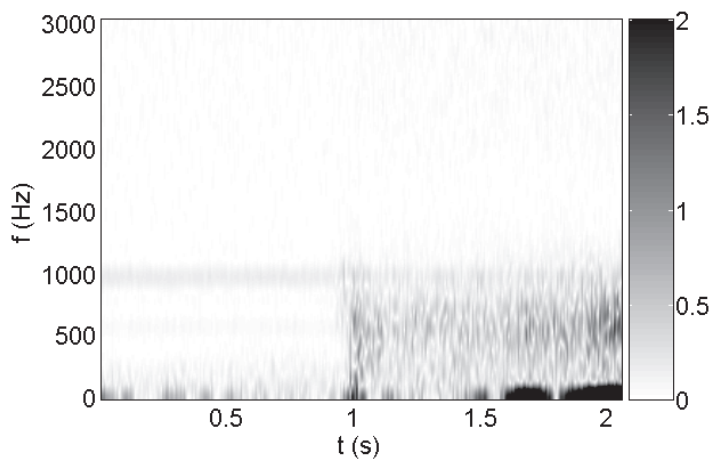

(f) $x / c=0.2, k=12$

Figure 14: Measurements, spectrograms from high frequency EMD fluctuation $\widetilde{C_{P}}(t)$ (Eq. 2) as a function of time for various transducers along the suction side, $\dot{\alpha}^{*}=0.18$ and $\alpha=0^{\circ}$ to $12^{\circ}, R e=750000$ 


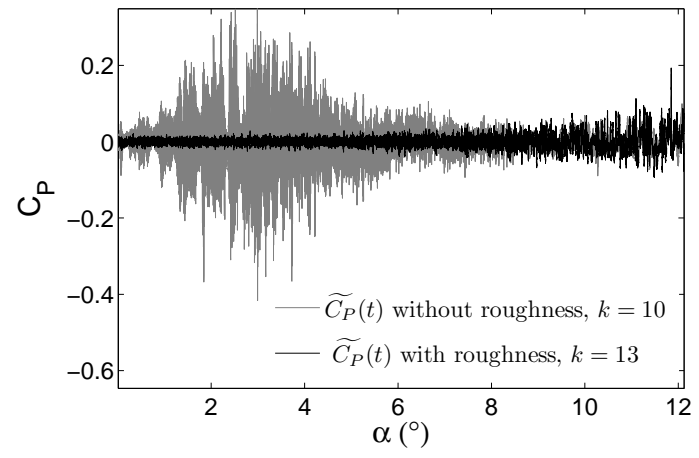

(a) $x / c=0.8$

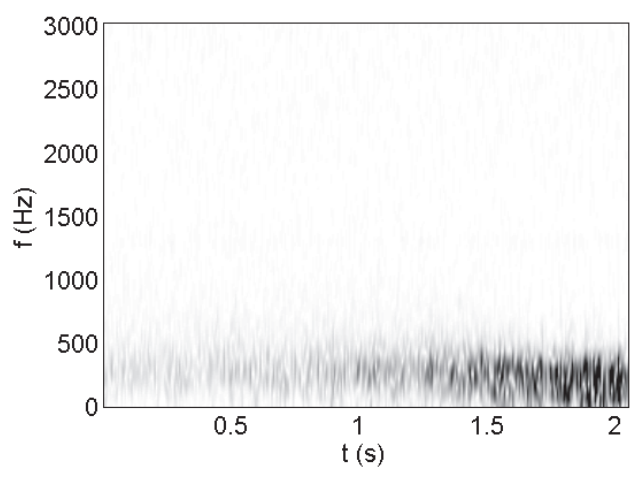

(c) $x / c=0.8, k=13$

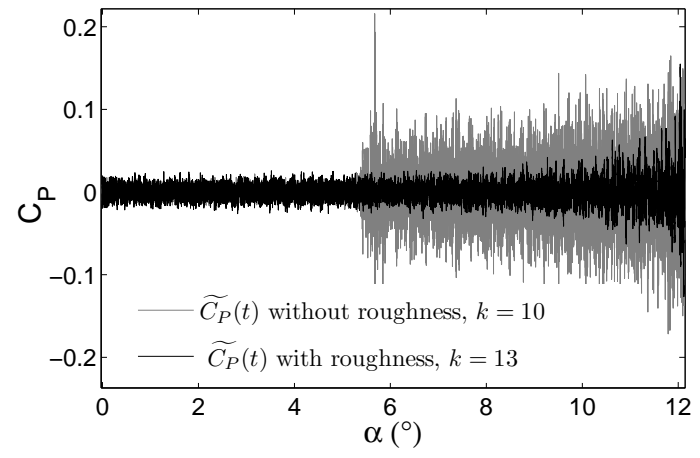

(b) $x / c=0.3$

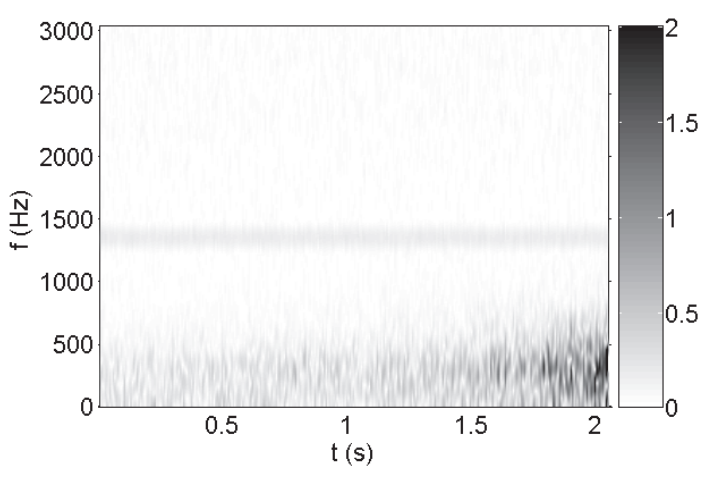

(d) $x / c=0.3, k=13$

Figure 15: Measurements, pressure fluctuations as function of the angle of incidence with and without roughness patch for $\dot{\alpha}^{*}=0.18, \alpha=0^{\circ} 12^{\circ}$ : (a) $x / c=0.8$, (b) $x / c=0.3$. Spectrograms from high frequency EMD pressure fluctuations $\widetilde{C_{P}}(t)$ (Eq. 2) as function of time, experiments with roughness patch: (c) $x / c=0.8$, (d) $x / c=0.3, R e=750000$ 


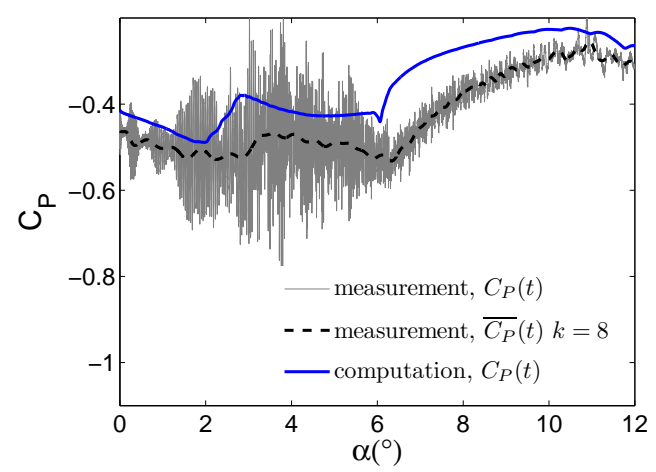

(a) $x / c=0.9$

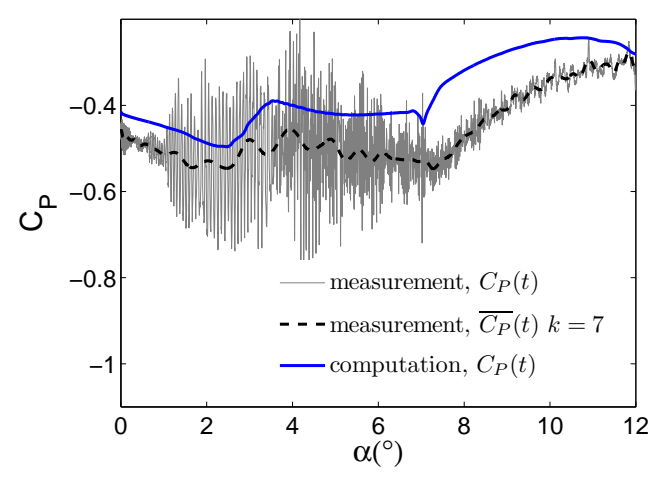

(c) $x / c=0.7$

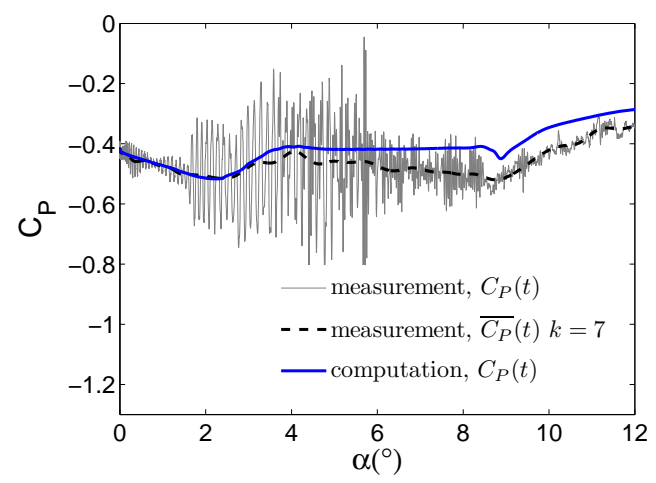

(e) $x / c=0.3$

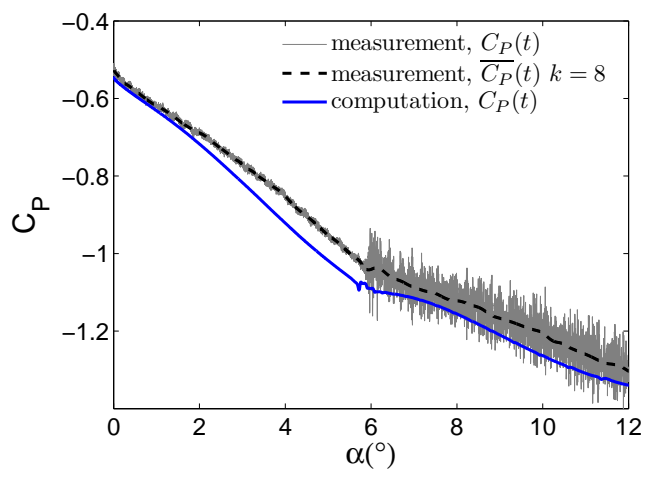

(b) $x / c=0.8$

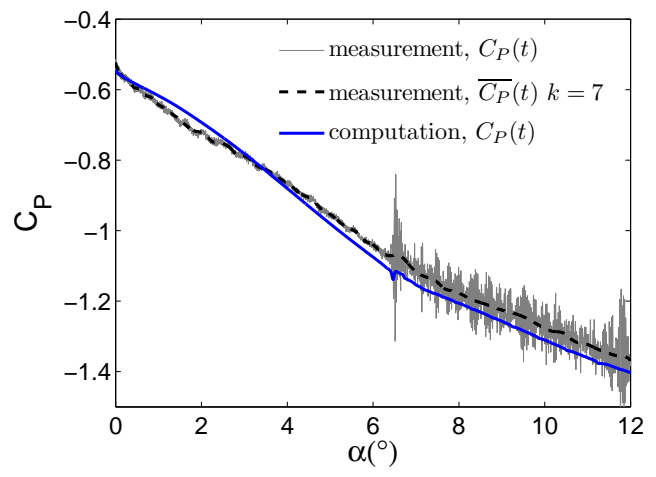

(d) $x / c=0.5$

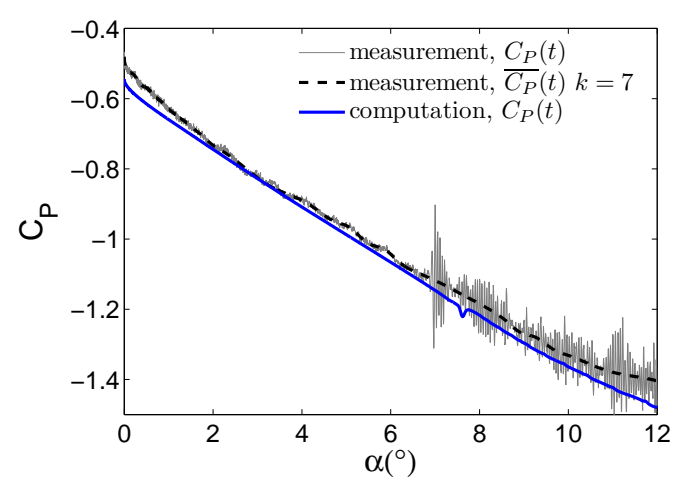

(f) $x / c=0.2$

Figure 16: Experimental and numerical pressure coefficient versus angle of incidence at $x / c=0.8$ and $x / c=0.3$ for various pitching velocities, $\alpha=0^{\circ}$ to $12^{\circ}$. The dotted lines are the low frequency EMD pressure signals $\overline{C_{P}}(t)$ (Eq. 1), $R e=750000$ 

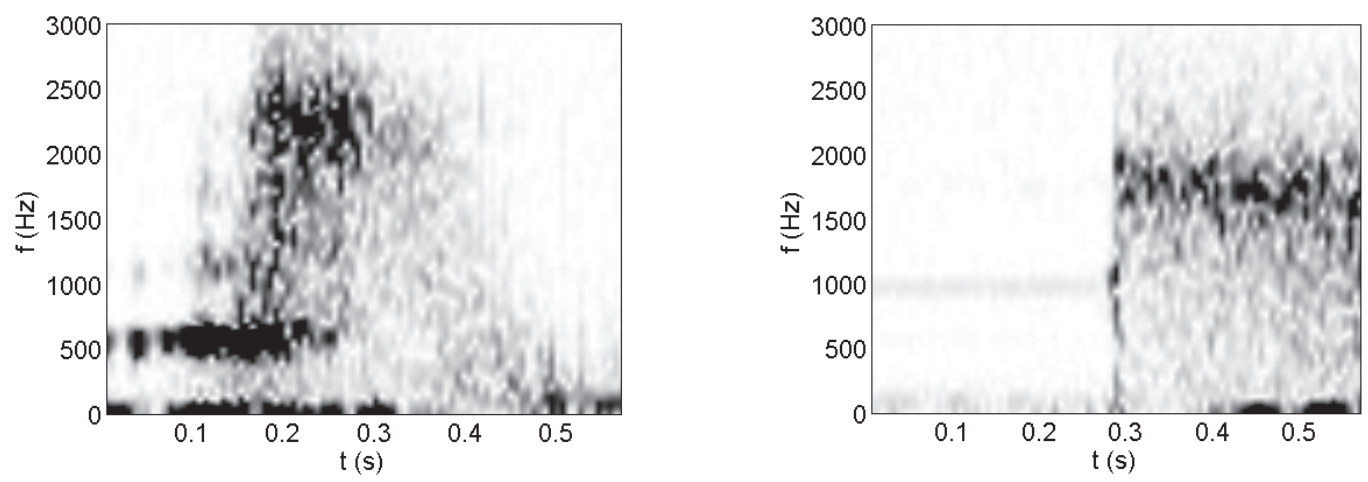

(a) $x / c=0.8, \dot{\alpha}^{*}=0.618, k=11$

(d) $x / c=0.3, \dot{\alpha}^{*}=0.618, k=11$
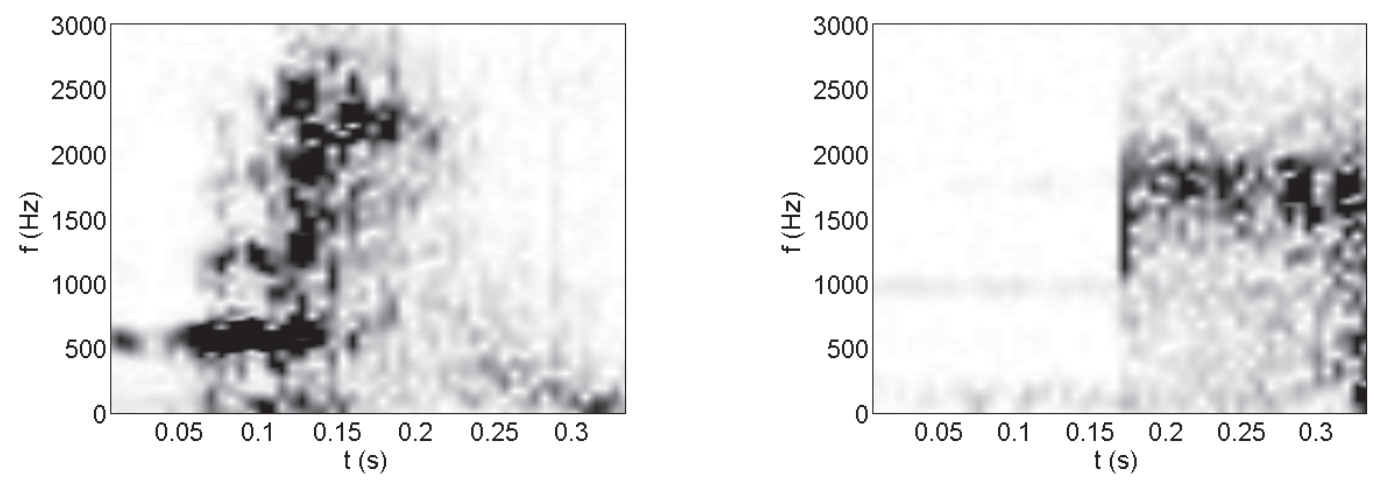

(b) $x / c=0.8, \dot{\alpha}^{*}=1.05, k=8$

(e) $x / c=0.3, \dot{\alpha}^{*}=1.05, k=8$
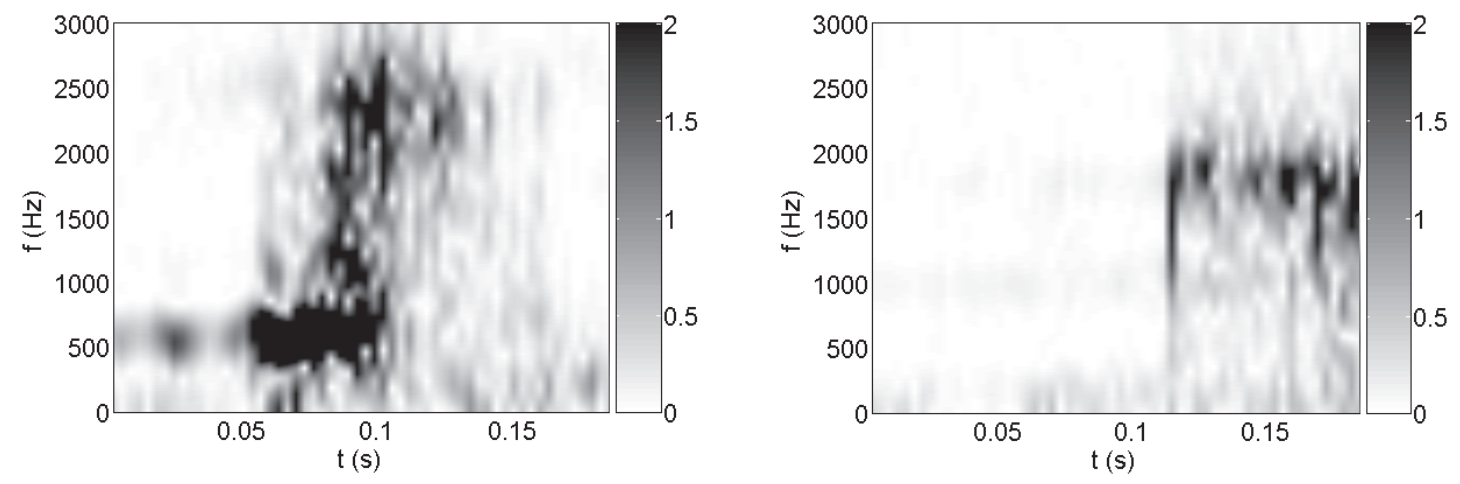

(c) $x / c=0.8, \dot{\alpha}^{*}=1.89, k=8$

(f) $x / c=0.3, \dot{\alpha}^{*}=1.89, k=8$

Figure 17: Measurements, spectrograms from high frequency EMD pressure fluctuations $\widetilde{C_{P}}(t)$ (Eq. 2) at $x / c=0.8$ and $x / c=0.3$ for various pitching velocities, $\alpha=0^{\circ}$ to $12^{\circ}$, $R e=750000$ 


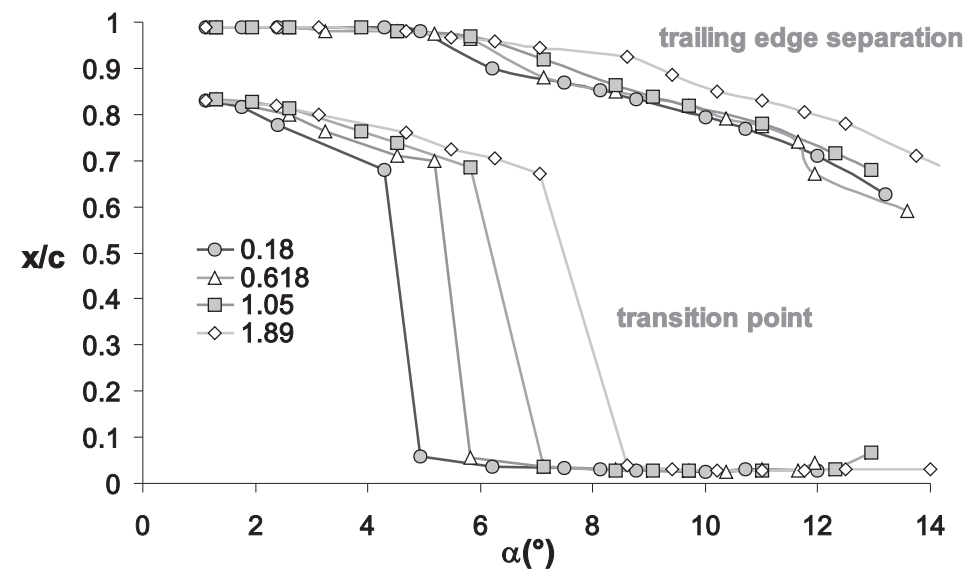

(a)

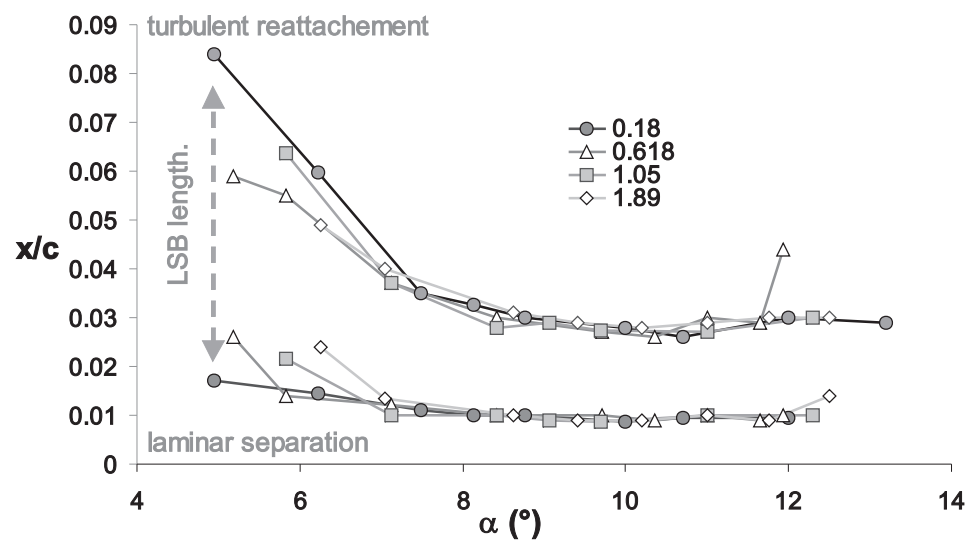

(b)

Figure 18: Numerical prediction of: (a) Boundary layer separation at leading edge and transition location on chord, (b) LSB length at leading edge on chord versus angle of incidence for various pitching velocities, $R e=750000$ 


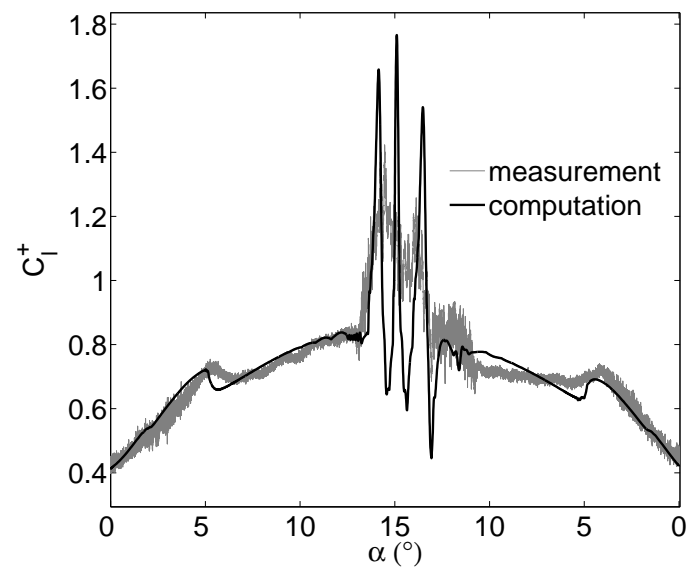

(a)

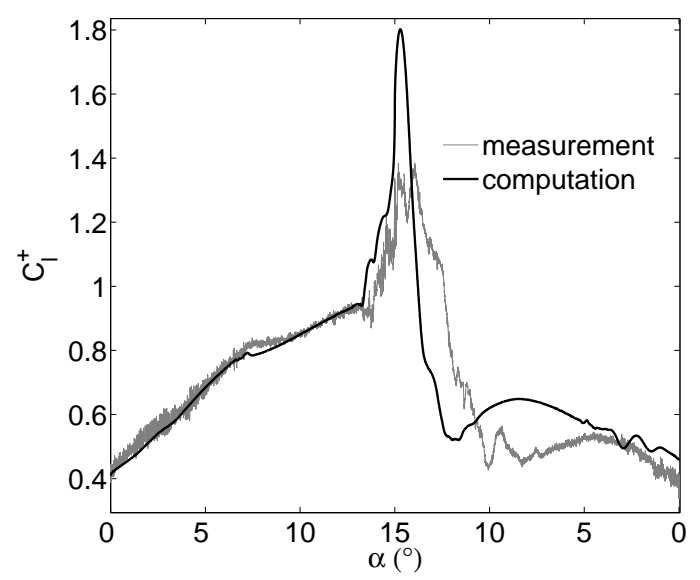

(c)

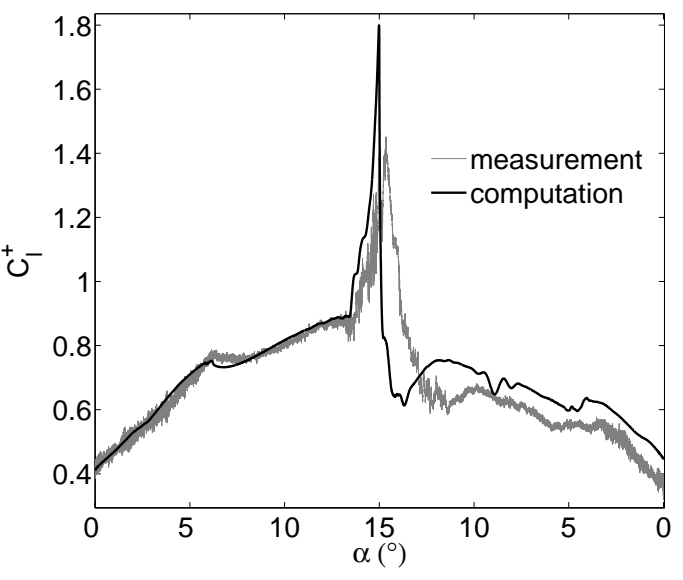

(b)

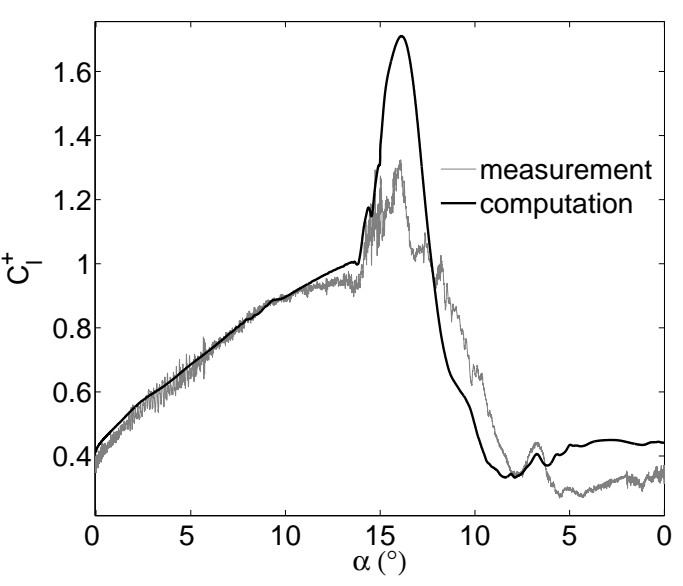

(d)

Figure 19: Measurements vs Computations, suction side loading versus angle of incidence during pitching motion: (a) $\dot{\alpha}^{*}=0.18$, (b) $\dot{\alpha}^{*}=0.618$, (c) $\dot{\alpha}^{*}=1.05$ and (d) $\dot{\alpha}^{*}=1.89, R e=750000$ 


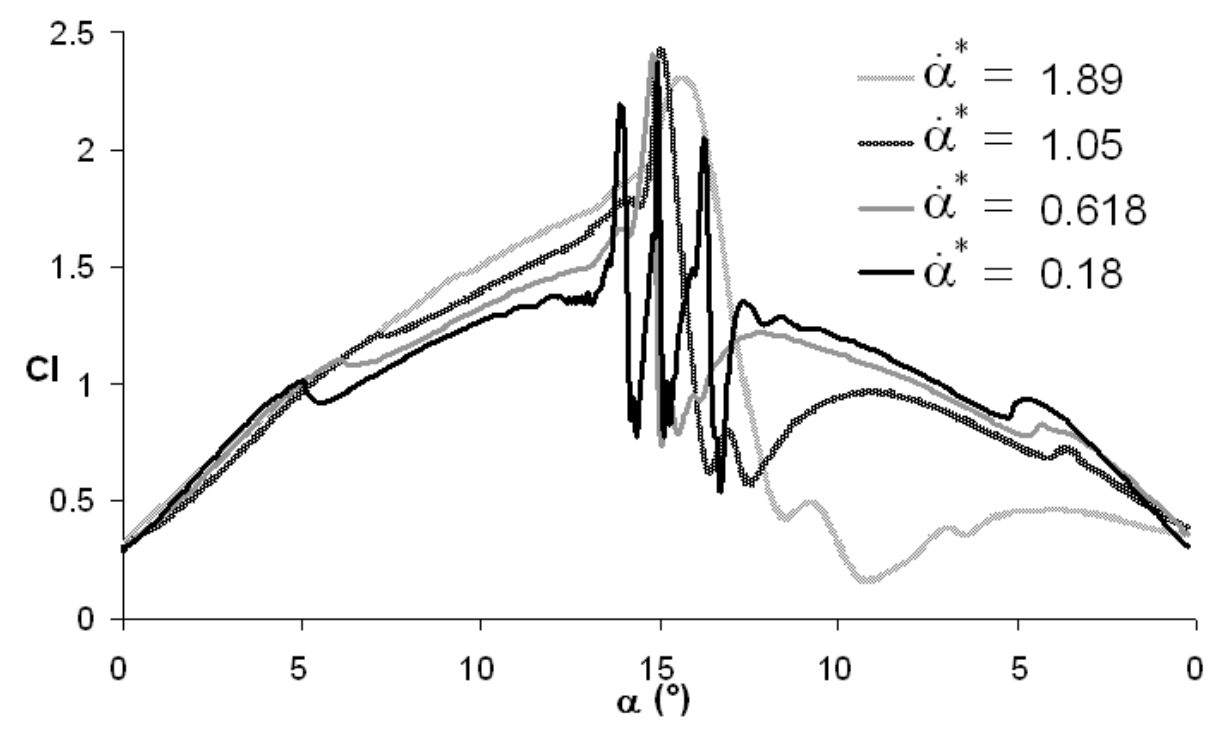

Figure 20: Computation, lift coefficient as a function of the angle of incidence for $\alpha=0^{\circ}$ to $15^{\circ}$ followed by the return to $0^{\circ}$, for 4 pitching velocities $\dot{\alpha}^{*}, R e=750000$ 


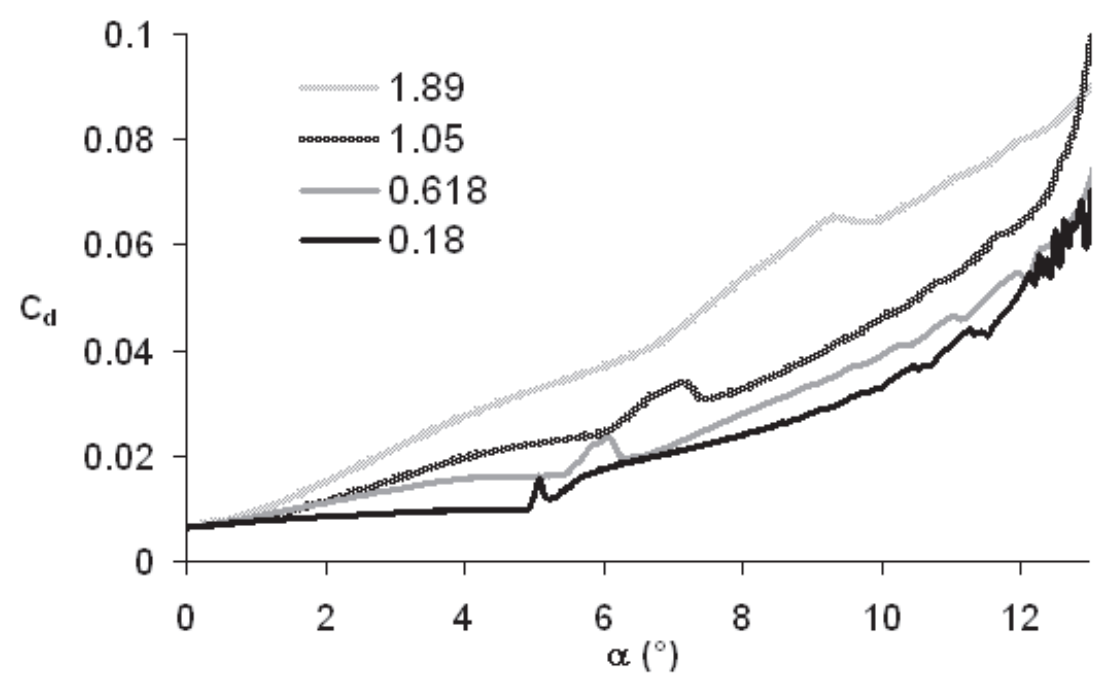

(a)

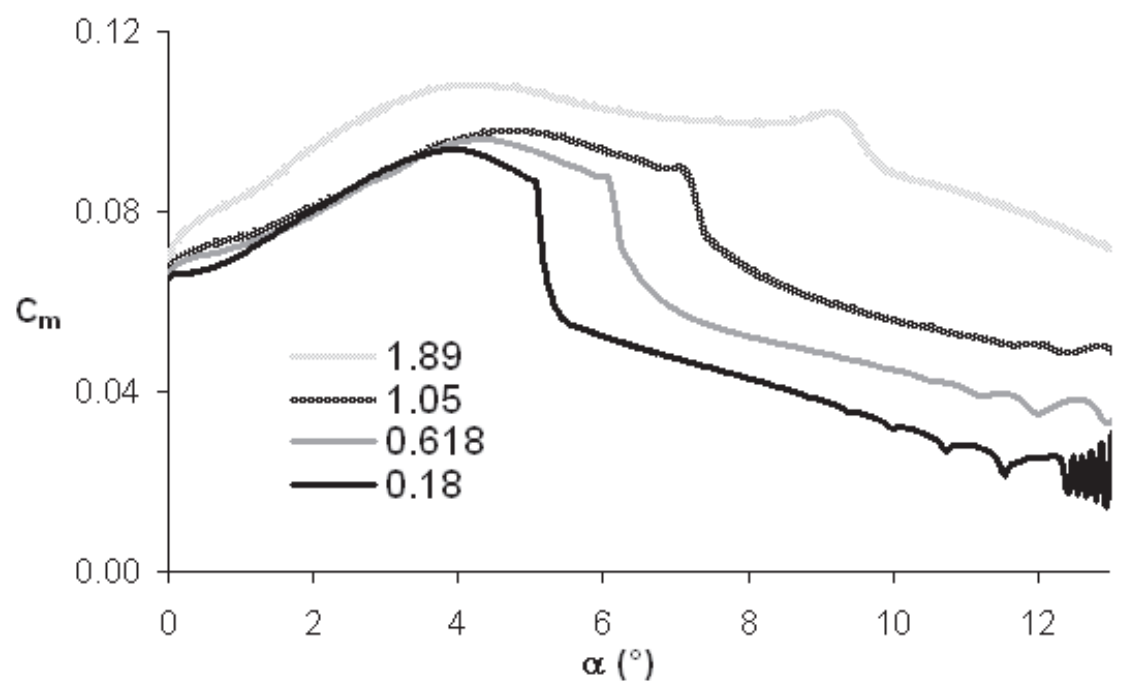

(b)

Figure 21: Computation, (a) drag coefficient and (b) moment coefficient versus angle of incidence for 4 pitching velocities $\dot{\alpha}^{*}, R e=750000$ 
Table 1: Computation,, lift and drag coefficients convergence according to boundary layer resolution, $\alpha=6^{\circ}, R e=750000$

\begin{tabular}{cccccc}
\hline Boundary layer resolution & $y^{+}$ & $C_{l}$ & $(\%)$ & $C_{d}$ & $(\%)$ \\
\hline \hline Wall function & 50 & 0.9661 & 3.3 & 0.0144 & 14.6 \\
\hline Low Reynolds & 2 & 0.9529 & 1.9 & 0.0163 & 3.2 \\
\hline Low Reynolds & 1 & 0.9503 & 1.6 & 0.0164 & 2.7 \\
\hline Low Reynolds & 0.5 & 0.9362 & 0.1 & 0.01676 & 0.5 \\
\hline Low Reynolds & 0.3 & 0.9353 & 0 & 0.01686 & 0 \\
\hline
\end{tabular}

Table 2: Computation, lift and drag coefficients as functions of number of elements $\alpha=6^{\circ}, R e=750000$

\begin{tabular}{cccccc}
\hline$N_{\text {foil }}$ & $N_{\text {total }}$ & $C_{l}$ & $(\%)$ & $C d$ & $(\%)$ \\
\hline \hline 50 & 18000 & 0.9915 & 4.3 & 0.02198 & 34.2 \\
\hline 100 & 23000 & 0.9545 & 0.4 & 0.01987 & 21.3 \\
\hline 200 & 40000 & 0.9477 & 0.3 & 0.01719 & 4.9 \\
\hline 400 & 66000 & 0.95026 & 0.1 & 0.0164 & 0.1 \\
\hline$N_{\text {max }}=800$ & 100000 & 0.95082 & 0 & 0.01638 & 0 \\
\hline
\end{tabular}

